\title{
.
}




\section{Developments in Cryoelectronics}

U.S. RTMENT OF MMERCE National D..reau C of o rds 153 630 172 
The National Bureau of Standards ${ }^{1}$ was established by an act of Congress March 3, 1901. The Bureau's overall goal is to strengthen and advance the Nation's science and technology and facilitate their effective application for public benefit. To this end, the Bureau conducts research and provides: (1) a basis for the Nation's physical measurement system, (2) scientific and technological services for industry and government, (3) a technical basis for equity in trade, and (4) technical services to promote public safety. The Bureau consists of the Institute for Basic Standards, the Institute for Materials Research, the Institute for Applied Technology, the Center for Computer Sciences and Technology, and the Office for Information Programs.

THE INSTITUTE FOR BASIC STANDARDS provides the central basis within the United States of a complete and consistent system of physical measurement; coordinates that system with measurement systems of other nations; and furnishes essential services leading to accurate and uniform physical measurements throughout the Nation's scientific community, industry, and commerce. The Institute consists of a Center for Radiation Research, an Office of Measurement Services and the following divisions:

Applied Mathematics-Electricity-Heat-Mechanics-Optical Physics-Linac Radiation ${ }^{2}$ - Nuclear Radiation ${ }^{2}$-Applied Radiation ${ }^{2}$ - Quantum Electronics ${ }^{3}$ Electromagnetics ${ }^{3}$-Time and Frequency ${ }^{3}$ - Laboratory Astrophysics ${ }^{3}$-Cryogenics ${ }^{3}$.

THE INSTITUTE FOR MATERIALS RESEARCH conducts materials research leading to improved methods of measurement, standards, and data on the properties of well-characterized materials needed by industry, commerce, educational institutions, and Government; provides advisory and research services to other Government agencies; and develops, produces, and distributes standard reference materials. The Institute consists of the Office of Standard Reference Materials and the following divisions:

Analytical Chemistry-Polymers-Metallurgy-Inorganic Materials-Reactor Radiation-Physical Chemistry.

THE INSTITUTE FOR APPLIED TECHNOLOGY provides technical services to promote the use of available technology and to facilitate technological innovation in industry and Government; cooperates with public and private organizations leading to the development of technological standards (including mandatory safety standards), codes and methods of test; and provides technical advice and services to Government agencies upon request. The Institute also monitors NBS engineering standards activities and provides liaison between NBS and national and international engineering standards bodies. The Institute consists of a Center for Building Technology and the following divisions and offices:

Engineering Standards Services-Weights and Measures-Invention and Innovation-Product Evaluation Technology-Electronic Technology-Technical Analysis-Measurement Engineering-Fire Technology-Housing Technology ${ }^{4}$ -Federal Building Technology ${ }^{4}$-Building Standards and Codes Services ${ }^{4}$ Building Environment ${ }^{4}$-Structures, Materials and Life Safet $y^{4}$-Technical Evaluation and Application ${ }^{4}$.

THE CENTER FOR COMPUTER SCIENCES AND TECHNOLOGY conducts research and provides technical services designed to aid Government agencies in improving cost effectiveness in the conduct of their programs through the selection, acquisition, and effective utilization of automatic data processing equipment; and serves as the principal focus within the executive branch for the development of Federal standards for automatic data processing equipment, techniques, and computer languages. The Center consists of the following offices and divisions:

Information Processing Standards-Computer Information-Computer Services -Systems Development-Information Processing Technology.

THE OFFICE FOR INFORMATION PROGRAMS promotes optimum dissemination and accessibility of scientific information generated within NBS and other agencies of the Federal Government; promotes the development of the National Standard Reference Data System and a system of information analysis centers dealing with the broader aspects of the National Measurement System; provides appropriate services to ensure that the NBS staff has optimum accessibility to the scientific information of the world, and directs the public information activities of the Bureau. The Office consists of the following organizational units:

Office of Standard Reference Data-Office of Technical Information and Publications-Library-Office of International Relations.

${ }^{1}$ Healquarters and Laboratories at Gaithersburg, Maryland, unless of herwise noted ; mailing ardress Washington, D.C. 20234

2 Part of the Center for Radiation Research.

3 Located at Boulder, Colorado 80302 . 
R.A. Kamper

D.B. Sullivan

Cryogenics Division

Institute for Basic Standards

U.S. National Bureau of Standards

Boulder, Colorado 80302

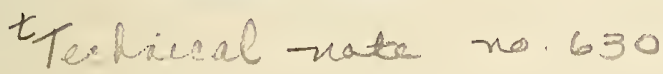

NBS Technical Notes are designed to supplement the Bureau's regular publications program. They provide a means for making available scientific data that are of transient or limited interest. Technical Notes may be listed or referred to in the open literature.

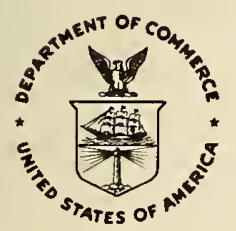

U.S. DePARTMENT OF COMMERCE, Peter G. Peterson, Secretary NATIONAL BUREAU OF STANDARDS, Lawrence M. Kushner, Acting Director Issued November 1972 
National Bureau of Standards Technical Note 630

Nat. Bur. Stand. (U.S.), Tech. Note 630, 73 pages (November 1972)

CODEN:NBTNAE

For sale by the Superintendent of Documents, U.S. Government Printing Office, Washington, D.C. 20402 (Order by SD Catalog No. C13.46:630.) 
$\underline{\text { Page }}$

SUMMARY .

1. BACKGROUND: SUPERCONDUCTIVITY, JOSEPHSON JUNCTIONS AND SQUIDS . . . . . . . . . . . . 3

2. MAGNETOMETRY . . . . . . . . . . . . 18

3. COMPUTERS . . . . . . . . . . . . . . . 24

4. FAR INFRARED TECHNOLOGY . . . . . . . . . 26

5. PRECISE METROLOGY . . . . . . . . . . . . 31

6. SPECIALIZED SCIENTIFIC INSTRUMENTS . • • • . . 44

7. TRANSMISSION LINES FOR INFORMATION • • • • . 51

8. ACCELEROMETERS AND GYROS . . . . . . . . . 53

9. CONCLUSION . • • • • • • • • • • • . • 55

References . . . . . . . . . . . . . . . . 56

\section{$\underline{\text { List of Figures }}$}

Figure 1. Practical Josephson junctions . . . . . . . 5

Figure 2. Current versus voltage characteristic of a thin film Josephson junction . • • . . . . . . . . 8

Figure 3. Dependence of $I_{c}$ (fig. 2) on applied magnetic field. . 9 Figure 4, The quantum interferometer . . . . . . . . 10 Figure 5. Variants of the SQUID . . . . . . . . . 12

Figure 6. Current versus voltage characteristic of a Josephson junction irradiated at a frequency $f=2 \mathrm{eV} / \mathrm{h}$. . . 13

Figure 7. Josephson oscillation . . . . . . . . . . 14

Figure 8. A magnetoca rdiogram obtained with a SQUID gradiometer . . . . . . . . . . 20 
List of Figures (continued)

Page

Figure 9. Comparison of measurements of e/h (1971). • . 30

Figure 10. Meservey's proposed absolute ammeter . . . . . 34

Figure 11. Circuit for precise dc measurements of current or voltage by counting flux quanta. . . . . . 36

Figure 12. Circuit for rf measurements using the flux quantum as a reference . . . . . . . . . . 40

Figure 13. Circuit for noise thermometry . . . . . . . 48 
Developments in Cryoelectronics

Robert A. Kamper and Donald B. Sullivan

\section{SUMMARY}

This is a survey of progress to date in the development of new electronic instruments taking advantage of the unique properties of superconductors. This has received copious attention in universities, and government and industrial laboratories, largely because of its strong appeal to physicists. Commercial activity has been in the nature of an investment in the future: very few devices have actually been sold yet. Computers, information transmission lines, and magnetocardiography may become commercially significant applications of superconductivity. Many other devices perform specialized functions of measurement and detection uniquely well, and will repay the cost of development without generating a large market. Josephson Junctions (weak but stable electrical contacts between superconductors), and the Superconducting QUantum Interference Devices (SQUIDs) derived from them, will probably have a significant impact on the following activities:

Magnetometry: including magnetocardiography, ELF communication, geomagnetism, and submarine detection. The SQUID is the world's most sensitive magnetic sensor. The first few commercial superconducting instruments have been SQUID magnetometers.

Computers: the tunneling cryotron is cheap, compact, suitable for mass production, and capable of sub-nanosecond switching times with extremely small dissipation of power.

Electrical Metrology: including maintaining the legal volt and measurement of current, voltage, power, and attenuation ratio, from dc through the microwave range of frequency.

Far Infrared: and millimeter wave receivers, harmonic mixers, and spectrum analyzers.

Galvanometers: null detectors, amplifiers, thermometers, phonon generators and detectors, etc. Specialized instruments for specialized scientific measurements.

* Contribution of the National Bureau of Standards, not subject to copyright. 
In addition, the simple properties of superconducting materials, such as the Meissner effect and the drastic reduction of electrical dissipation, can be used to make very significant improvements in the performance of the following devices:

Information Transmission Lines: superconducting coaxial lines combine physical compactness with a wide bandwidth and low attenuation.

Pulse Delay Lines: require the same qualities as for transmission of information.

Stable Oscillators: recent developments in the stabilization of microwave oscillators, with superconducting resonant cavities, have created the world's most stable oscillator over periods of time up to a few seconds. Combined with the available long-term stability of the cesium beam resonator, this will become the best oscillator over any period of time.

Accelerometers: magnetic levitation using the Meissner effect, and superconducting magnets operating in the persistent current mode, give a very stable and almost frictionless suspension. This has proven worth for the measurement of long-term variations in gravitation, and possible usefulness for gyros. detail.

The remainder of this report will discuss these topics in greater

Key Words: Electronics; Josephson effect; precise measurements; superconductivity. 


\section{BACKGROUND: SUPERCONDUCTIVITY, JOSEPHSON JUNCTIONS AND SQUIDS}

Superconductors comprise a class of metals which undergo a reversible, thermodynamic phase transition at a low critical temperature $T_{c}$ which is characteristic of the individual material. At temperatures above $T_{c}$ they have no common characteristic to set them apart from other metals. Below $T_{c}$ they enter a state characterized by the vanishing of electrical resistance to small direct currents. The highest known value of $\mathrm{T}_{\mathrm{c}}$ is $21 \mathrm{~K}$ for an alloy of niobium, aluminum and germanium. The superconductors commonly used in devices have transition temperatures ranging from $3 \mathrm{~K}$ to $18 \mathrm{~K}$. These include the elements tin $(3.7 \mathrm{~K})$, lead $(7.2 \mathrm{~K})$, and niobium $(9.4 \mathrm{~K})$; the alloys niobium-titanium $(6 \mathrm{~K}-10 \mathrm{~K})$, and lead-tin $(7 \mathrm{~K}-8 \mathrm{~K})$; and the intermetallic compounds $\mathrm{Nb}_{3} \mathrm{Sn}(18 \mathrm{~K})$ and $\mathrm{NbN}(16 \mathrm{~K})$. Superconducting devices normally operate in a bath of liquid helium, to mainta in the low temperature required for the superconducting state.

Although the electrical resistance of superconductors is strictly zero only for direct current, it remains exceptionally low for frequencies from zero through the microwave range. At the higher frequencies it becomes a sensitive function of the preparation of the surface of the superconductor.

Another striking feature of the superconducting state, discovered by Meissner and Ochsenfeld in 1933, is known as the Meissner Effect. It would occur in its simplest form in a perfect, defect-free, single crystal of a superconducting metal with a smooth, regular shape. If such a specimen were to be cooled through its transition temperature in the presence of a small magnetic field, the magnetic flux would be completely expelled from its interior, to return again on warming 
through $T_{c}$. This is a thermodynamically reversible effect which cannot be explained without considerations that go beyond the vanishing of electrical resistance.

The situation in real specimens of superconducting metals is complicated by the tendency of irregularities of structure or composition to impede the motion of the flux lines, so that in practice one usually observes only a partial exclusion of flux. Another complication is the concentration of magnetic flux at sharp corners of the specimen, leading to very large flux densities in small regions. These are accommodated by partial penetration of magnetic flux into a structure of finely divided superconducting and normal state domains, known as the intermediate state. For this reason experiments are usually performed with rod-shaped specimens oriented parallel to the applied magnetic field.

The Meissner effect is the consequence of electric currents which flow spontaneously on the surface of the specimen or on the boundary between the superconducting and normal state phases. The current density falls off approximately exponentially with depth below the surface of the superconductor, with a characteristic length $\lambda$ known as the penetration depth. The magnitude of $\lambda$ depends upon the composition of the superconductor, and the temperature and magnetic field at the surface. It is typically of the order of $10^{-7}$ to $10^{-8} \mathrm{~m}$. The magnetic flux also penetrates to this depth below the surface of the superconductor.

Josephson junctions are weak, stable electrical contacts between superconductors $[1-3]^{1}$. Several practical ways of making them are illustrated in figure 1. An ideal junction might consist of two super-

${ }^{1}$ Figures in brackets indicate the literature references at the end of this paper. 


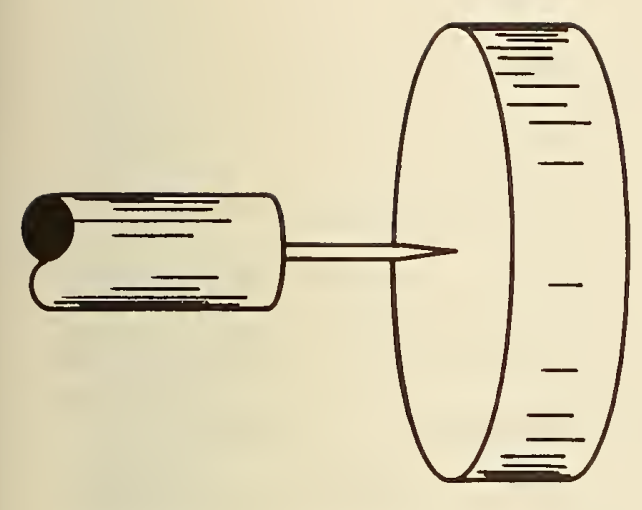

Ð

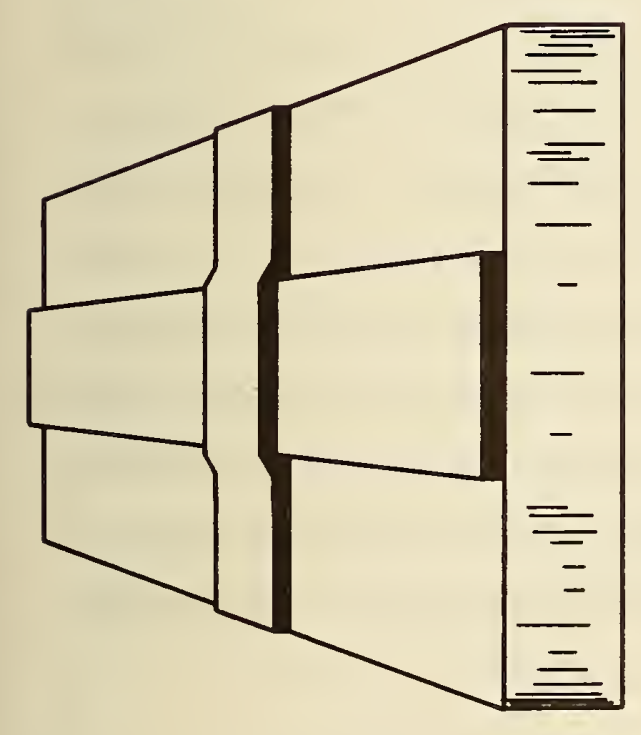

$\vec{\sigma}$
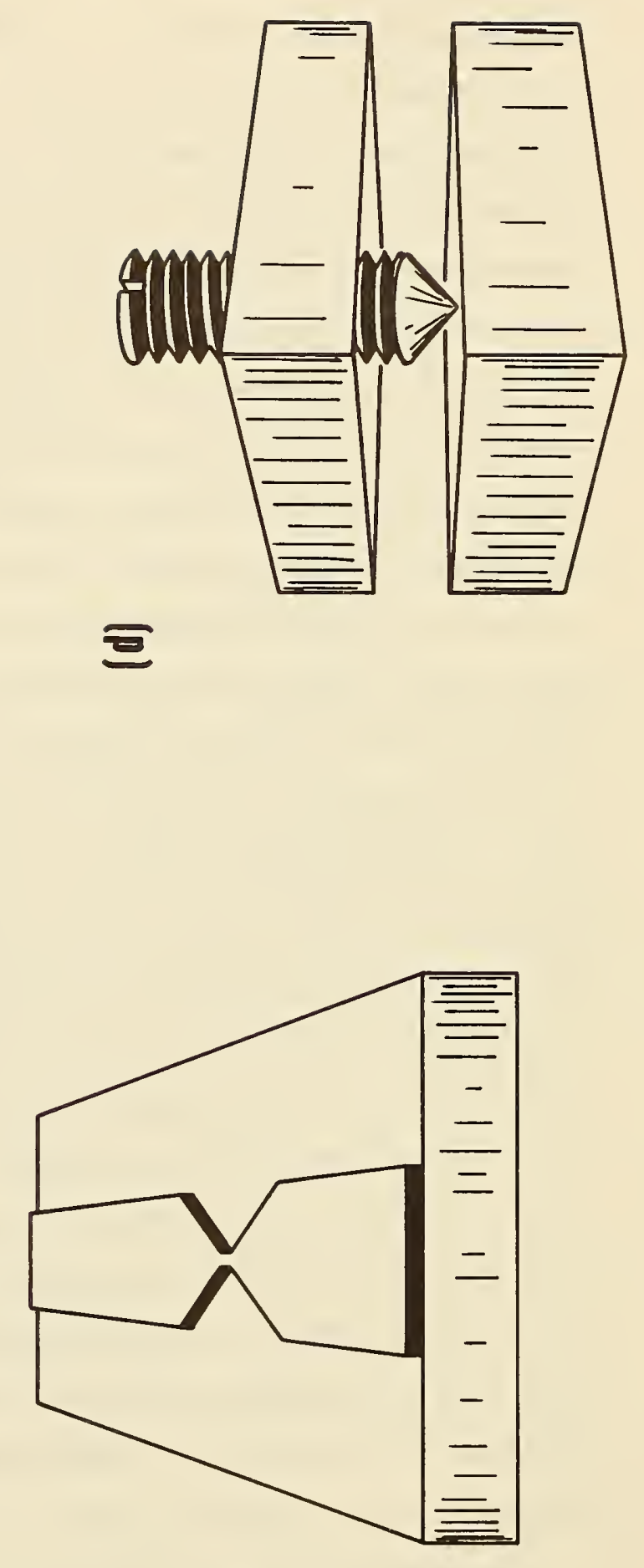

5
0
0
3
0
0

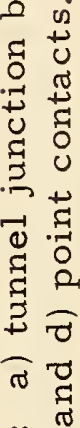

i⿱ $)$

品

㝴

룽 द 00

क

U)

0 으

च

出 党

0

荕

-1

$\Xi$ 
conductors separated by a vacuum gap about $10 \AA\left(10^{-7} \mathrm{~cm}\right)$ wide and perhaps a square millimeter in area. Electrons would cross this gap by the quantum mechanical tunnel effect [4]. The nearest practical approach (fig. la) consists of a strip of thin film of one superconductor deposited on an insulating substrate such as glass, quartz, or sapphire. Its surface is covered with an insulating barrier of the desired thickness (usually by oxidation of the metal), and then a strip of thin film of the second superconductor is deposited across the first to complete the junction. The barrier does not need to be an insulator. A layer of normal metal such as copper, about 10,000 $\AA$ thick, will work instead [5]. Another approach (fig. 1b) is to use a continuous strip of superconducting thin film with a very narrow constriction [6] (a few micrometers wide). The electrical characteristics of this microbridge structure differ from those of crossed film junctions, but the similarities are sufficient for many practical purposes. An intermediate approach (fig. lc,d) is to press a slender point on one superconductor lightly against the other to form a point contact junction [7]. It is not quite clear whether a surface layer formed by atmospheric corrosion forms an insulating barrier, or if the narrow contact area functions as a microbridge. However, point contacts have the great advantage that they can be adjusted while in operation and they work very well. The more permanent structures such as crossed film junctions and microbridges have the compensating advantage of greater mechanical stability. The relative merits of tunnel junctions, microbridges, and mechanical point contacts (insofar as they are understood at this writing) were spelled out at length at the 1972 Applied Superconductivity Conference $[8-11]$.

Superconductors commonly used to make Josephson junctions are lead, tin, indium, niobium, tantalum, etc. It is not important whether the same or two different superconductors are used to form a junction. 
The electrical characteristics of these junctions depend on how they are made [12-14], but one feature they all have in common is the ability to pass a limited current (in the range from a few microamperes to a few milliamperes) without any voltage appearing. A somewhat idealized I-V curve for a crossed film junction is shown in figure 2. If the current passing through the junction exceeds $I_{c}$ (fig. 2) a voltage appears and there is often an unstable region of negative dynamic resistance. There is a characteristic voltage of the order of a millivolt, determined by the energy gaps of the superconductors, at which the current rises sharply before settling down to an ohmic response at high voltage. Microbridges differ from crossed film junctions mainly by the absence of the low current region at intermediate voltage.

The magnitude of $I_{c}$ is very sensitive to a magnetic field applied in the plane of the junction. Figure 3 shows a plot of $I_{c}$ versus magnetic flux density for an ideal crossed film junction. Similar curves have been observed in the laboratory [15]. The vertical scale depends critically on the thickness of the insulating barrier, while the horizontal scale is controlled by the width of the junction and the properties (penetration depths) of the superconductors. The periodicity is caused by quantum interference between electrons crossing different parts of the barrier. It is analogous to optical diffraction at a narrow slit. The analog of optical interference between two slits can be created by connecting two Josephson junctions in parallel in a superconducting circuit as shown in figure 4 . The curve shown in figure 3 then becomes the envelope of an interference pattern of much shorter period. This period corresponds to the quantum of magnetic flux $\mathrm{h} / 2 \mathrm{e} \approx 2 \times 10^{-15}$ weber (h is Planck's constant and $e$ is the electron charge). Each maximum in the interference pattern occurs when a whole number of magnetic 


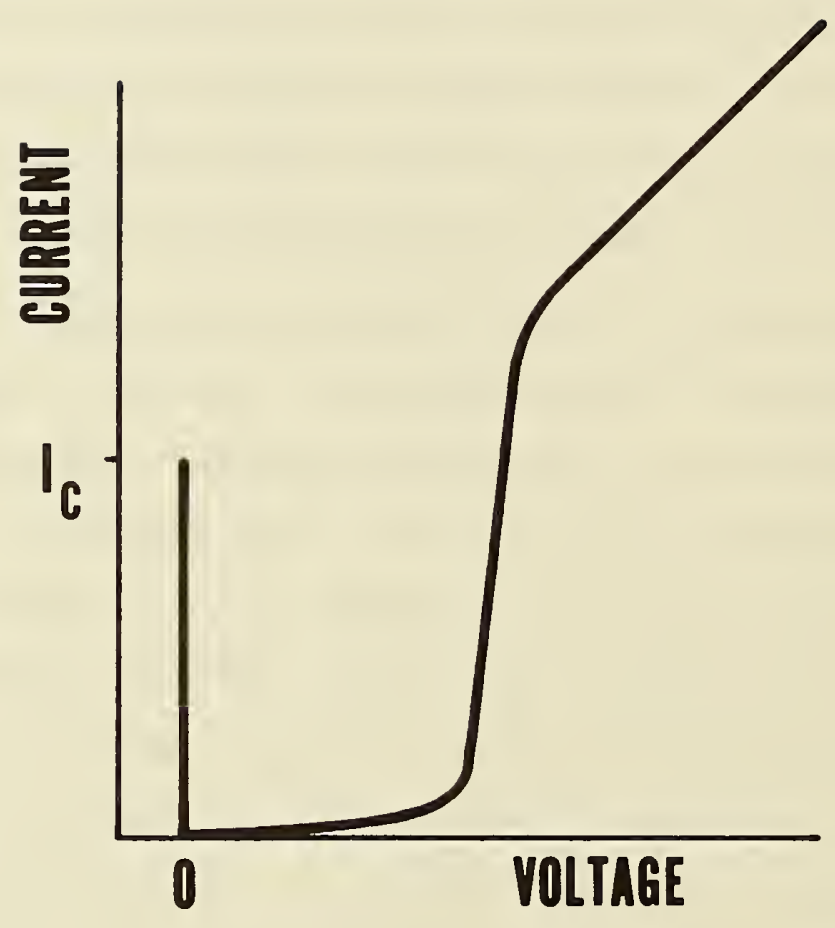

Figure 2. Current versus voltage characteristic of a thin film Josephson junction. 


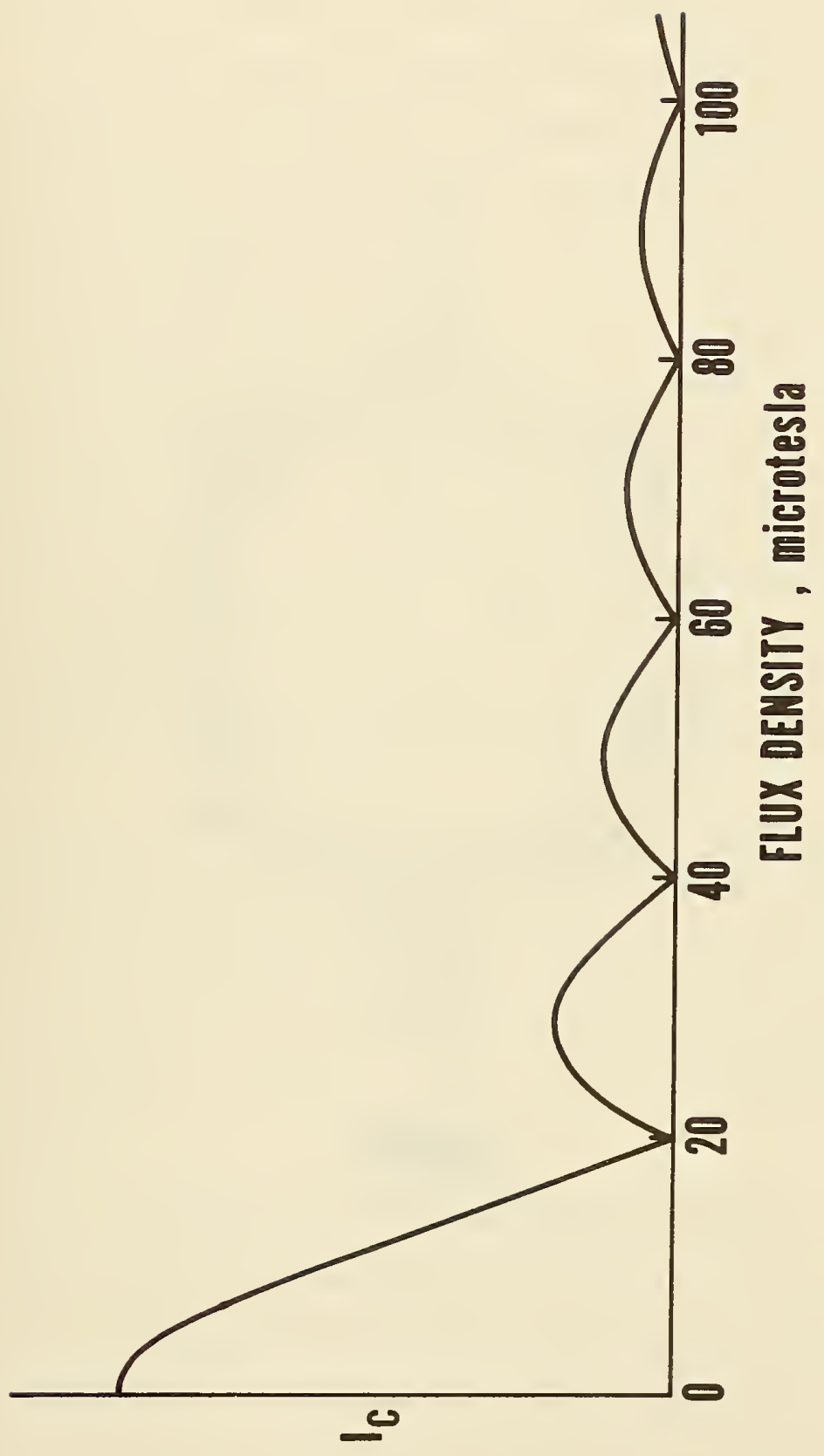

孚

렁

․

$\stackrel{\circ}{\circ} \frac{0}{0}$

$m$
0
2
5
0
0
-1
5 


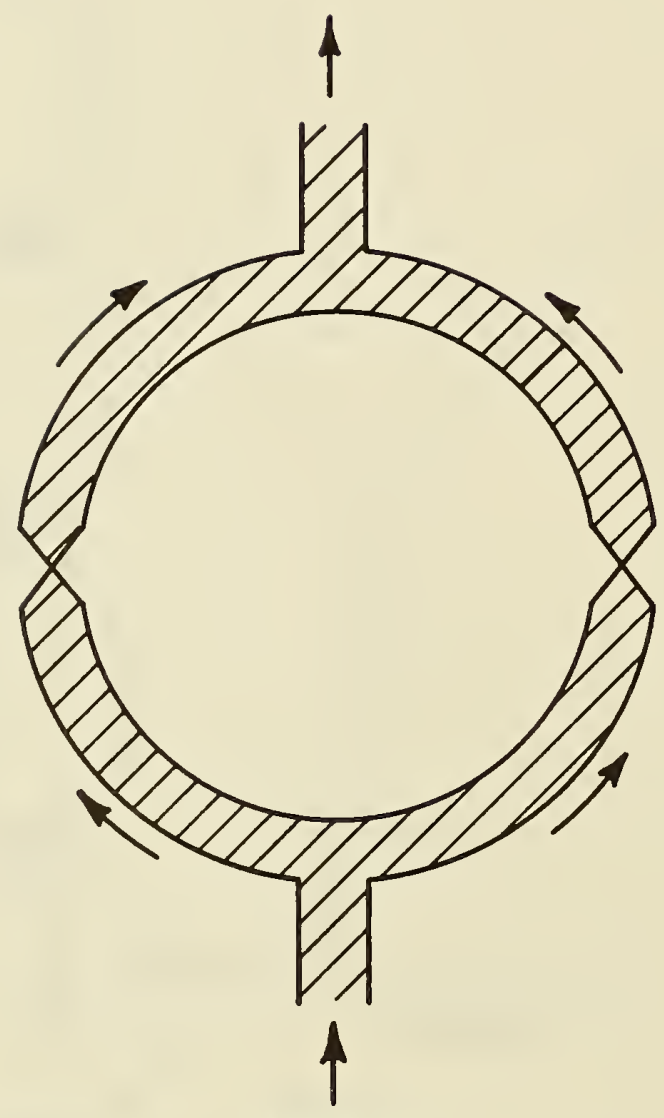

CURRENT

Figure 4. The quantum interferometer. 
flux quanta link the superconducting loop. This arrangement has become known as a quantum interferometer [16]. It has obvious applications to magnetometry and related fields .

It is possible to observe the effects of magnetic flux quantization with a single Josephson junction closing a superconducting loop, by monitoring the impedance of the loop at a convenient radio frequency. The resulting device, known as the rf-biased Superconducting QUantun Interference Device (SQUID) has received intensive development as a magnetic sensor [17-21]. Several versions of the SQUID are illustrated in figure 5. The most advanced design is the one at lower right. It consists of 12 superconducting loops (with very small mutual inductance) connected in parallel across a single point-contact Josephson junction [22]. This gives the device great sensitivity to variations in magnetic flux and also a strong readout signal. This latter can also be improved by raising the readout frequency $[17,23]$. Silver and Zimmerman have written an exhaustive discussion of all aspects of SQUIDs [24].

If a radio frequency or microwave signal is addressed to a bare Josephson junction, the direct current versus voltage relationship becomes profoundly modified as shown in figure 6. It develops a series of steps at equal intervals of voltage [25]. The voltage interval $\mathrm{V}$ is related to the frequency $f$ by $h f=2 \mathrm{eV}$. This corresponds to approximately $484 \mathrm{MHz} / \mu \mathrm{V}$.

Finally, if a receiver is connected to the junction a coherent oscillation [25-27] can be detected at the same frequency $f=2 \mathrm{eV} / \mathrm{h}$. The waveform is usually nonsinusoidal, so harmonics of this frequency can also be detected at submultiples of the fundamental voltage. These are illustrated by an actual recorder chart shown in figure 7 . The receiver was tuned to $43 \mathrm{MHz}$ and the odd signal shape was a result of 


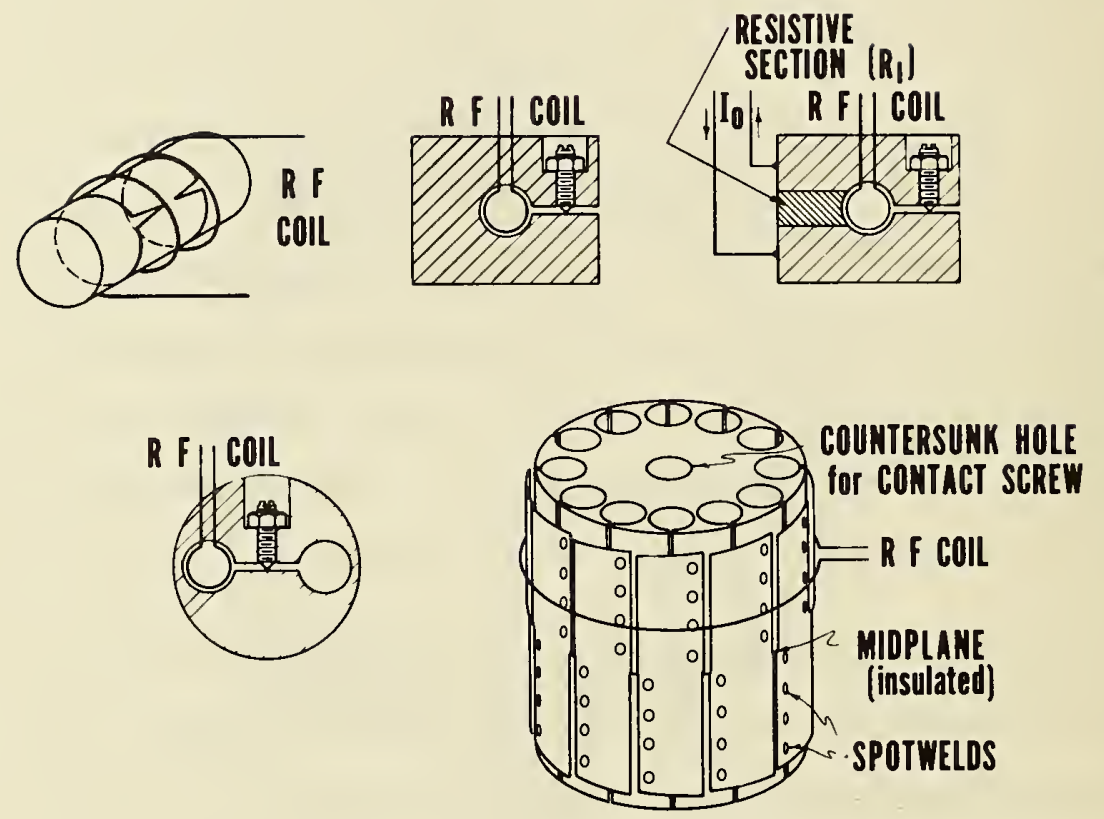

Figure 5. Variants of the SQUID. 


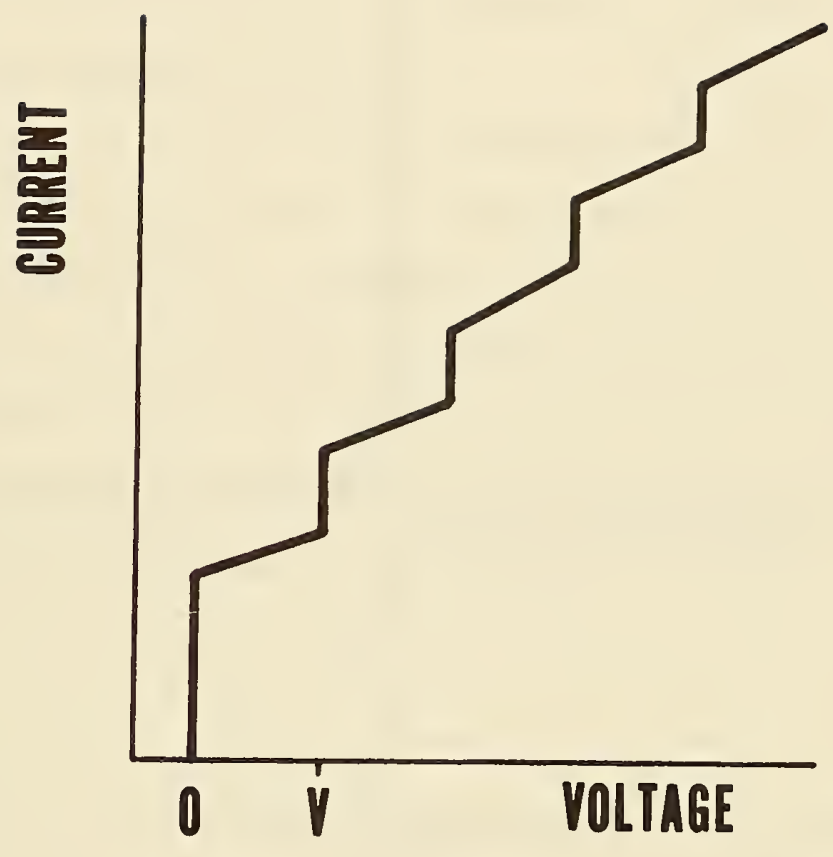

Figure 6. Current versus voltage characteristic of a Josephson junction irradiated at a frequency $f=2 \mathrm{eV} / \mathrm{h}$. 


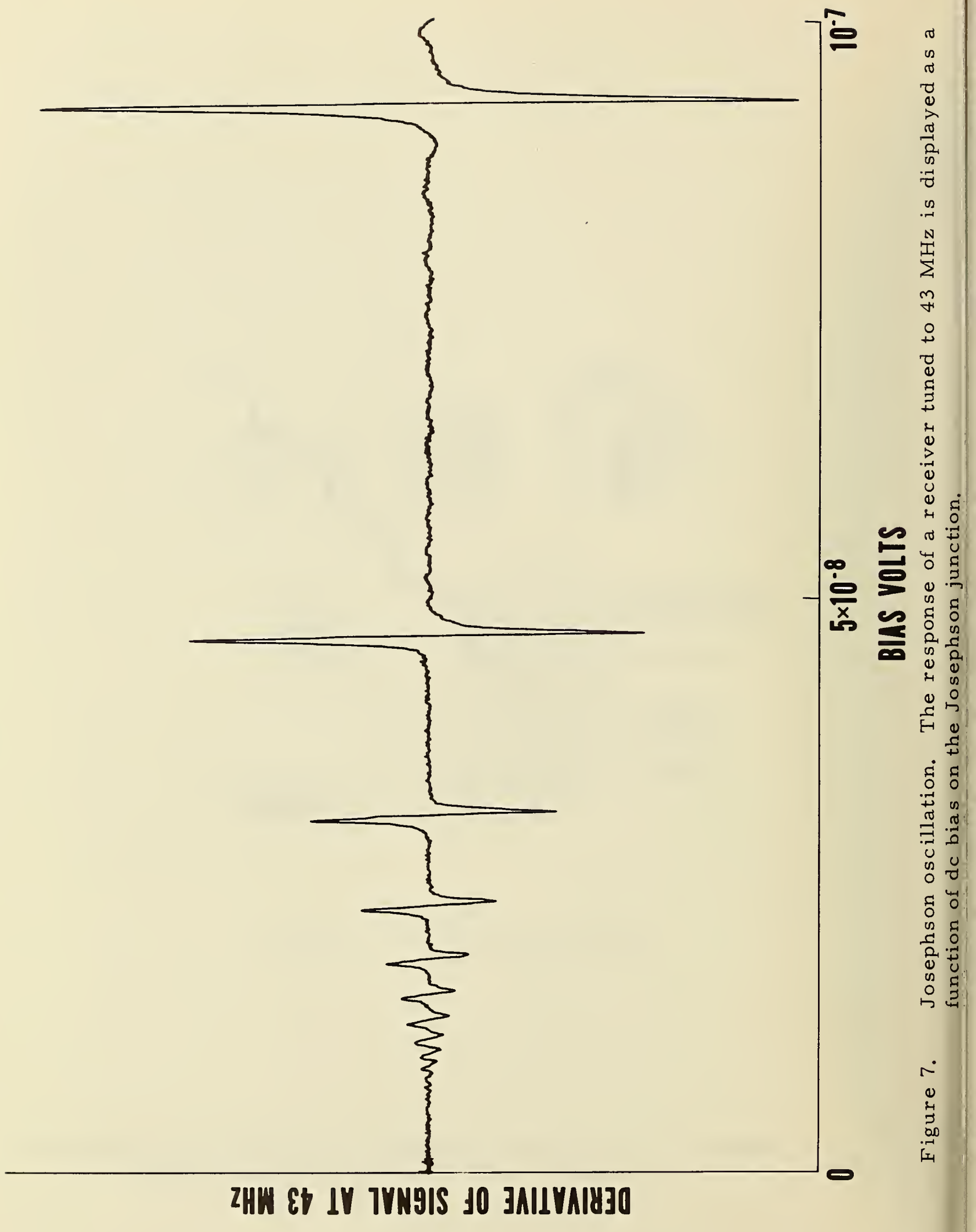


lock-in detection. Frequencies ranging from a fraction of $1 \mathrm{~Hz}$ [28] to $270 \mathrm{GHz}$ [29] have been observed. The presence of oscillation at frequencies up to $8 \mathrm{THz}$ has been inferred indirectly [30].

The Josephson effect is a quantum mechanical phenomenon on an unusually large scale. It happens because the superconducting state is one in which the conduction electrons of the metal have a high degree of order. The wave functions describing their motion have a coherence of phase extending over large distances--greater than a meter in one observation [31]. Variations in the phase of these wave functions control the movements of the electrons and hence the currents which flow in the superconductor. A Josephson junction is a place where the wave function is sufficiently weak to permit a discontinuity of phase, but not weak enough to interrupt communication between the superconductors or either side completely. Thus a "supercurrent" (a dissipationless current) can flow across the junction. The magnitude $J$ of the current density depends upon the quantum mechanical phase difference $\theta$ thus

$$
J=J_{0} \sin \theta
$$

where $J_{0}$ is a constant depending on the properties of an individual junction. If a voltage $\mathrm{V}$ is applied across the junction, the phase difference $\theta$ advances so that its rate of change is

$$
d \theta / d t=4 \pi e V / h .
$$

Equations (1) and (2) were originally derived from fundamental quantum mechanics by Josephson [1]. Together they are known as the Josephson equations. Taken together they account for all the bizarre properties of point contact Josephson junctions and microbridges. Combining them, we find that the supercurrent I flowing through a single point contact obeys the equation 


$$
\mathrm{dI} / \mathrm{dt}=(2 \mathrm{eV} / \mathrm{h}) \sqrt{\mathrm{I}_{\mathrm{c}}^{2}-\mathrm{I}^{2}}
$$

where $I_{c}$ is defined in figure 2. In addition to the supercurrent obeying equation (3), there is an ohmic leakage current and a displacement cur rent through the capacitance of the junction which modify its electrical characteristics. This behavior leads directly to such functions as microwave mixing, harmonic generation, oscillation, and the constant voltage steps induced in the dc characteristics by irradiation with microwaves. These last come about because of the combined functions of harmonic generation and self-oscillation. Whenever the voltage is such that the frequency of self-oscillation coincides with one of the harmonics of the incoming microwaves, the two signals phase lock together to generate a stable zero-frequency beat which contributes to the steady current flowing through the junction.

The phenomenon of interference which is observed in extended or multiple junctions in the presence of a magnetic field is a consequence of the fact that a magnetic field induces a phase shift in the wave function. The total phase shift $\theta$ integrated around a closed loop of superconductor linked by a magnetic flux $\varphi$ is

$$
\theta=4 \pi e \varphi / h
$$

If the loop contains two Josephson junctions this phase difference will appear across them. When it is equal to $\pi, 3 \pi, 5 \pi$, etc., the phase differences across the two junctions must be equal and opposite. Hence, according to equation ( 1 ), the currents flowing through the two junctions must be equal and opposite also. This occurs when $\varphi=h / 4 \mathrm{e}, 3 \mathrm{~h} / 4 \mathrm{e}$, $5 \mathrm{~h} / 4 \mathrm{e}$, etc. For intermediate values of magnetic flux (and hence phase difference) the two currents add. This is closely analogous to optical 
interference of light transmitted by a pair of slits. Inspection of equations (1) and (4) will also show that the response of a single extended Josephson junction to a magnetic field, shown in figure 3 , is a nalogous in the same way to optical diffraction by a single slit. In a.solid superconducting ring, with no Josephson junctions, the total phase shift must be $0,2 \pi, 4 \pi$, etc., for the wave function to be single valued. Hence $\varphi$ is constrained to be an integral multiple of $h / 2 \mathrm{e}$. This is the phenomenon of magnetic flux quantization. 


\section{MAGNETOMETRY}

The most sensitive magnetometers existing today consist of SQUIDs coupled to the field to be sensed via superconducting flux transformers. It is possible to dispense with the SQUID by mechanically modulating the inductance of a superconducting circuit to generate an audio-frequency signal proportional to the linked magnetic flux. However, none of the instruments which have been built on this principle can compete with SQUIDs in sensitivity, so we dismiss them with a reference to reviews by Opfer [32] and Deaver and Goree [33].

One of the earliest descriptions of a properly engineered SQUID magnetometer was published by Forgacs and Warnick [34]. Taking advantage of the periodic nature of the basic response of the SQUID, they used two modes of operation. In the first, or analog mode, the magnetic flux linking the SQUID was modulated at an audio frequency, and the output of the readout circuit was detected with a lock-in detector with reference derived from the modulation. The output of the lock-in detector was amplified and supplied to a coil coupled to the SQUID, so as to servo the flux linking the SQUID to remain constant in the face of variations in the external field. The current required to do this is linearly related to the external field, and was used to drive the output indicator of the instrument. In the second, or digital mode, an up-down counter was used to count the flux quanta driven through the SQUID by variations in the external field. The counter was driven by two lock-in detectors, one operating at the modulation frequency and the other at the second harmonic thereof. This combination supplied sufficient information to give the counter a sense of direction. This early instrument did not use a flux transformer, relying instead on the direct coupling of the (double junction) SQUID to the external field. 
Subsequent improvements in magnetometry have been directed towards improvements in the SQUID to increase the signal-to-noise ratio, and hence the sensitivity and bandwidth (permitting faster counting). Also, rf-biased SQUIDs appear to have inherently greater dynamic range than the double junction type, which suffers from the need for an adjustable $(\mathrm{dc})$ bias level to cover a range of more than a few hundred flux quanta. Many millions of quanta can be sensed by an rf-biased single junction SQUID with no adjustment of the operating conditions. Sensitivity of the order of $10^{-16} \mathrm{~T} / \sqrt{\mathrm{Hz}}$ is now available with an instrument developed by Zimmerman and Frederick [35]. This instrument has a multiple-loop SQUID operating at a readout frequency of $300 \mathrm{MHz}$.

Using this sensitivity to the best advantage is another problem. The natural fluctuations in the Earth's magnetic field are of the order of $10^{-11} \mathrm{~T}$ in the range of frequency used in magnetometry. Two alterna tives are open: the full sensitivity of a SQUID magnetometer may be used in a heavily shielded enclosure, or the external coils of the flux transformer may be arranged to convert the instrument to a magnetic gradiometer. This is done by using two equal coils wound in opposition and placed a finite distance apart. With careful attention to balancing the device the magnetic flux coupled to the SQUID by the pair of coils in response to a uniform external field can be nulled out. A gradient in the external field will permit coupling of the difference in flux linking the coils to the SQUID. The result is to make the instrument insensitive to disturbances from distant sources, but to retain its full sensitivity to signals from sources at a distance comparable to the separation of the coils.

The development of gradiometers has occupied the attention of several groups of researchers, because they are the most useful form of ultrasensitive magnetometers. The major problem has been the 


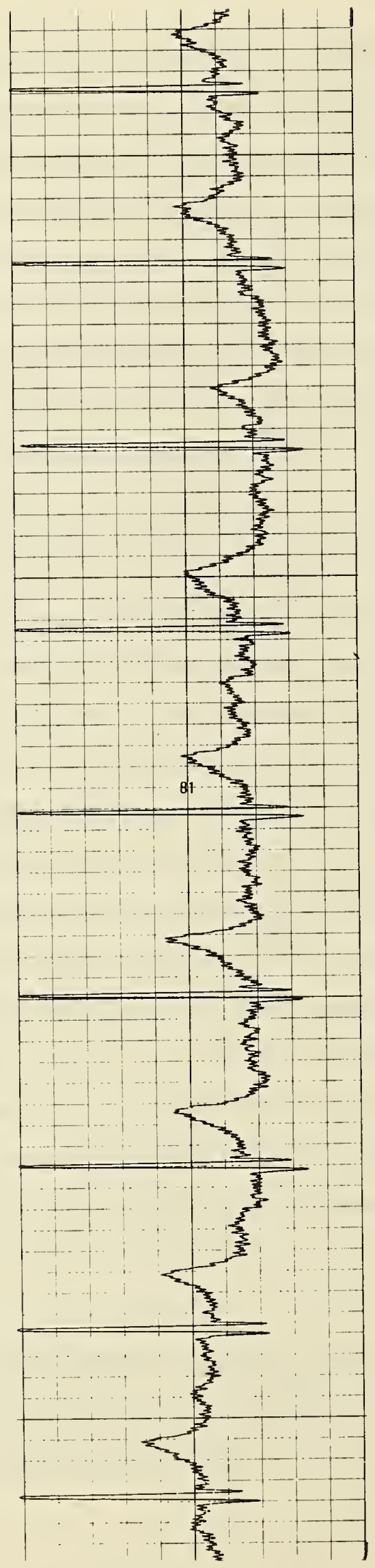

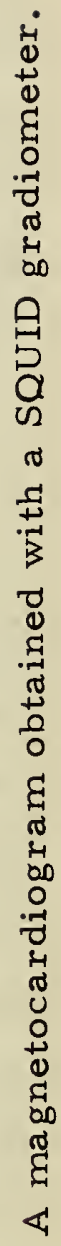

$\underset{1}{\infty}$ 
surprising difficulty in attaining a stable balance. Typically gradiometers have appeared to be unbalanced by as much as several percent, and often the unbalance changes unpredictably between measurements. Usually it is greater than expected from the precision with which the coils were made. Although it is not fully understood, the origin of this unbalance appears to be the movement of trapped magnetic flux in the superconducting metal of the flux transformer. The cure was discovered by Zimmerman and Frederick [35]. It consists of the simple strategy of making the flux transformer with many turns of very fine superconducting wire, to severely limit the freedom of the trapped flux to migrate. This enabled them to achieve a stable balance of one part in $10^{5}$ (both axial and transverse). This is just sufficient to enable them to use the full sensitivity of their best instrument while exposed to the natural fluctuations of the Earth's field, away from magnetic objects which generate local gradients.

The most promising applications of ultrasensitive magnetometers (at the time of writing) are the following:

Magnetocardiography

The electrical activity associated with the action of a beating heart drives small electric currents in the torso which can be detected with a very sensitive magnetometer. The resulting record bears a strong resemblance to an electrocardiogram (fig. 8). Whether it contains more or less information is an open question at present, to be answered by research which is now in progress. The main operational advantage of magnetocardiography is freedom from the need to attach electrodes to the patient. The main inconvenience is the need for heavy magnetic shielding to isolate the weak cardiac signal from the background noise of a hospital. This need may be softened by further sophistication in signal processing. 
The pioneering work in magnetocardiography was done by Baule and McFee [36] without the aid of an ultrasensitive magnetometer. They used a large coil of wire connected to a conventional amplifier to pick up the weak signal. It was necessary to use an electrocardiac signal for a phase reference to extract the wanted magnetic signal from the background noise. Subsequently, Cohen [37], at the National Magnet Laboratory, built an elaborate magnetic shield and used a SQUID magnetometer to obtain unassisted magnetocardiograms of similar quality to standard electrocardiograms. He is now the acknowledged leader in this field of research. Two reports of magnetocardiograms obtained with gradiometers instead of magnetic shielding have appeared in the literature $[23,38]$. Both required "quiet" locations away from electrical machinery. In the light of Cohen's most recent work [39], the future of magnetocardiography looks very bright if it wins acceptance by the medical profession.

Magnetoencephalography

There is also magnetic activity in the brain. The expected signal levels are even lower than those generated by the heart. A signal at the frequency of the $\alpha-$ rhythm $(\sim 10 \mathrm{~Hz})$ has been detected by Cohen [40], with a coil of wire and conventional amplifier, using a signal from electrodes on the subject's head to supply the phase information required for signal averaging. Several groups intend to explore this field with SQUID magnetometers, but not much has been accomplished yet.

Magnetic Anomaly Detection

There is some activity and speculation on the detection of magnetic anomalies (e.g., submarines) with sensitive magnetometers. For this, SQUIDs would be used in the gradiometer configuration. The work has not yet proceeded far enough to demonstrate its value. 


\section{$\underline{\text { ELF Communication }}$}

Matching requirements force antennas, for radio reception at extremely low frequencies, to become inconveniently large if they are to retain acceptable efficiency with normal resistive materials. Below a frequency of about $100 \mathrm{~Hz}$ it is generally conceded that a sensitive magnetometer would be a better receiver than a conventional loop antenna and radio receiver. Once again SQUTDs would offer the extra possibility of the gradiometer configuration.

ELF communication is useful in situations where the signal must traverse a path of significant length through water or rock. The conductivity of these preferentially attenuates electromagnetic fields at high frequencies. One suggested need for ELF communication is the location of trapped miners after an underground disaster, and another is for communication with submarines.

\section{Commercial Activity}

We are aware of a few cornpanies, mostly small, who are selling SQUID magnetometers with various degrees of sophistication. Total sales probably amount to about 250 at the time of writing. It is possible to buy the basic SQUID and electronic readout equipment plus flux trans formers, etc., on a custom basis, or a combined magnetometer and superconducting shield for magnetic measurements on materials, particularly rock specimens. Equipment suitable for magnetocardiography is also under commercial development. 


\section{COMPUTERS}

Both the logic and the memory of a computer are built up with switches, which are required in enormous numbers. The desirable qualities of these switches are:

1) they must be cheap,

2) they must be suitable for simultaneous fabrication in compact arrays with a high yield,

3) they must dissipate very little heat,

4) they must switch very quickly, and

5) their electrical characteristics must be compatible with a physically realizeable network to convey the pulses which actuate them from one to another.

The story of superconducting computers began with Buck's invention of the Cryotron in 1956 [41]. This was a switch in which a superconducting "gate" (originally a fine wire, later a strip of thin film) was switched from the superconductive to the resistive state by the magnetic field from a current in the "control": a wire winding or strip of thin film in close proximity to the gate. The control was also made of superconducting material. The gain of the cryotron was defined as the ratio of the critical current of the gate to the control current required to switch the gate to the resistive state. If this is greater than unity cryotrons can be cascaded in complex logic circuits without the need for intermediate amplifiers.

The invention of the cryotron created a flurry of activity to solve some of the problems it presented, notably in the technology of thin evaporated films (it quickly became apparent that a version made of thin films would be superior to the original wire-wound device). This in turn stimulated the development of other thin film devices, both for logic 
and for memory [42]. However, judged by the criteria listed at the beginning of this section, none of these devices (including the cryotron itself) could compete successfully with the advancing semiconductor and magnetic core devices. The development of all of them has been abandoned, with the exception of a single survivor.

The survivor is the Tunneling Cryotron, invented by Matisoo at IBM in 1967 [43]. It differs from the thin film version of Buck's cryotron in that the gate is replaced by a Josephson tunnel junction, which has much more desirable switching characteristics than any plain strip of superconducting film. Its principle of operation may be understood with reference to figure 2 , which shows the current versus voltage characteristics typical of the gate of this device. If it is supplied with a stabilized current less than $I_{c}$ there are two possible operating points: the first at zero voltage and the second on the steeply rising part of the curve at a voltage of the order of a millivolt. A very small magnetic field in the plane of the junction, generated by the control strip, will suffice to lower $I_{c}$ sufficiently to switch the device from the first operating point to the second (see fig. 3). A temporary interruption of the gate current will switch it back again. An individual junction will switch in $85 \times 10^{-12} \mathrm{sec}[44]$, requiring a pulse of energy of only $10^{-13}$ Joules [45]. Cycle times of $550 \times 10^{-12} \mathrm{sec}$ have been achieved for steering current from one limb of a network to another [44].

The tunneling cryotron is under active development. Its remaining problems appear to be soluble, and the chances are good that it will finally lead to the realization of a commercially viable superconducting computer. 


\section{FAR INFRARED TECHNOLOGY}

One of the present frontier areas in radio technology is the part of the electromagnetic spectrum at frequencies above $100 \mathrm{GHz}$. The development of infrared lasers has provided powerful, coherent sources of radiation throughout this range: for example at $981 \mathrm{GHz}(\mathrm{HCN}) ; 2.5$, 3.8 , and $10.7 \mathrm{THz}\left(\mathrm{H}_{2} \mathrm{O}\right)$; and at 28.3 and $32.2 \mathrm{THz}\left(\mathrm{CO}_{2}\right)$. Receivers and other signal processing equipment are not yet available to take full advantage of these lasers. For example, the best available receiver at $300 \mathrm{GHz}$ has a noise temperature of $2000 \mathrm{~K}$, and things get worse at higher frequencies. There is a clear need for new devices which the Josephson effect may be able to supply, at least in the first two decades of this range of frequency. The first significant work in this field was started by Grimes, Richards, and Shapiro [46,47] at the Bell Telephone Laboratories. The two topics which have attracted the greatest attention so far are detectors and harmonic mixers. Putting together the hardware that has been developed to make a proper receiver is probably one or two years away.

Far infrared detection is unusual in that even the best nonsuperconducting detectors operate at very low temperatures. The reason is that at frequencies below $10 \mathrm{THz}$ the quantum of radiation has less energy than $\mathrm{kT}$ at ambient temperature, so any process which would respond to such small photons would be subject to noisy thermal excita tion. A good example of the semiconductor detectors available in this spectral range is Low's germanium bolometer [48]. It has a noise equivalent power of $5 \times 10^{-13} \mathrm{~W} / \sqrt{\mathrm{Hz}}$ when operating at $2 \mathrm{~K}$.

An early superconducting device was a thin-film bolometer reported by Martin and Bloor in 1961 [49]. This consisted of a strip of thin superconducting film, on a very light substrate, connected to the helium bath via a thermal resistance. The temperature of the film 
would therefore be increased slightly by power absorbed from the incident radiation. When the equilibrium temperature of the film was adjusted to coincide with its transition to the resistive state, its resistance would be very sensitive to small changes in temperature caused by small changes in the level of irradiation. In its final form, this device had a noise equivalent power of $10^{-12} \mathrm{~W}$ with a time constant of $1.25 \mathrm{sec}$. It became the workhorse of a productive group of infrared spectroscopists at Queen Mary College in London [50]. At the low frequency end of the infrared spectral range, it is possible to do even better with a Josephson junction for a detector.

Radiation detection with a Josephson junction is based on the properties of the current versus voltage characteristic illustrated in figure 6. The constant-voltage steps in the curve appear under the influence of irradiation, so the current flowing through the junction when it is biased to a voltage near one of the steps is clearly a sensitive function of the intensity of the incoming radiation. The width $I$ (in current) of the nth step varies with the amplitude of the voltage $\mathrm{V}_{0}$, induced in the junction by the radiation, approximately according to function

$$
I_{n}=I_{c} J_{n}\left(2 e V_{o} / h f\right)
$$

where $f$ is the frequency of the radiation and $J_{n}$ is the nth order Bessel function. Since the spacing of the steps (in voltage) is proportional to the frequency of the radiation, the Josephson junction can be used as a tunable detector. Its sensitivity is enhanced by coupling it to a resonant cavity, also tuned to the frequency of the incoming radiation. The resulting detector was reported by Richards and Sterling [51] to have a noise equivalent power of less than $10^{-14} \mathrm{~W} / \sqrt{\mathrm{Hz}}$ at $190 \mathrm{GHz}$. This sensitivity remains close to the best that has been attained. The 
question of the high frequency limit of radiation detection by Josephson junctions has been addressed by McDonald, et al. [30,52-54]. They have observed the steps in the current versus voltage characteristic to extend to $17 \mathrm{mV}$, implying the presence of harmonically generated currents at frequencies up to $8.2 \mathrm{THz}$. They actually observed harmonic mixing (and hence detection) with a signal at $3.8 \mathrm{THz}$ from a water vapor laser.

Ulrich [55] has taken the first steps towards astronomy with a detector based on a Josephson junction. He has made some observations of the sun, moon, and Venus at a frequency of $250 \mathrm{GHz}$.

Harmonic generation and mixing is another function for which the Josephson junction is uniquely well adapted. It has the feature that the power converted into the harmonics of an incoming signal diminishes only slowly with increasing harmonic number, compared with other devices. McDonald, et al., have observed the generation of the 401 st harmonic of a signal at $9 \mathrm{GHz}$ from a klystron, and the difference signal created by mixing it with a signal from a water vapor laser at $3.8 \mathrm{THz}$ [54]. This performance is out of reach of any other known device.

Another mode of operation of a Josephson junction is to use its self oscillation for a local oscillator [56]. Often the output is taken at zero frequency, and this is essentially the mode in which Richards and Sterling's resonant detector operated [51]. With suitable electronics to locate incipient steps in the current versus voltage characteristics, a Josephson junction can be used as a quick-reading spectrometer throughout the range up to its high frequency limit $[30,57]$.

The first applications of far infrared and sub-millimeter wave receivers will probably be to astronomy. This spectral range is rich in molecular resonances which could give abundant information about 
conditions in various parts of the solar system. Coming closer to home, the same resonances can be used to detect pollutants in our atmosphere. Another, more distant, prospect is communication, over short distances in the atmosphere (because of attenuation) and over long distances in outer space. 


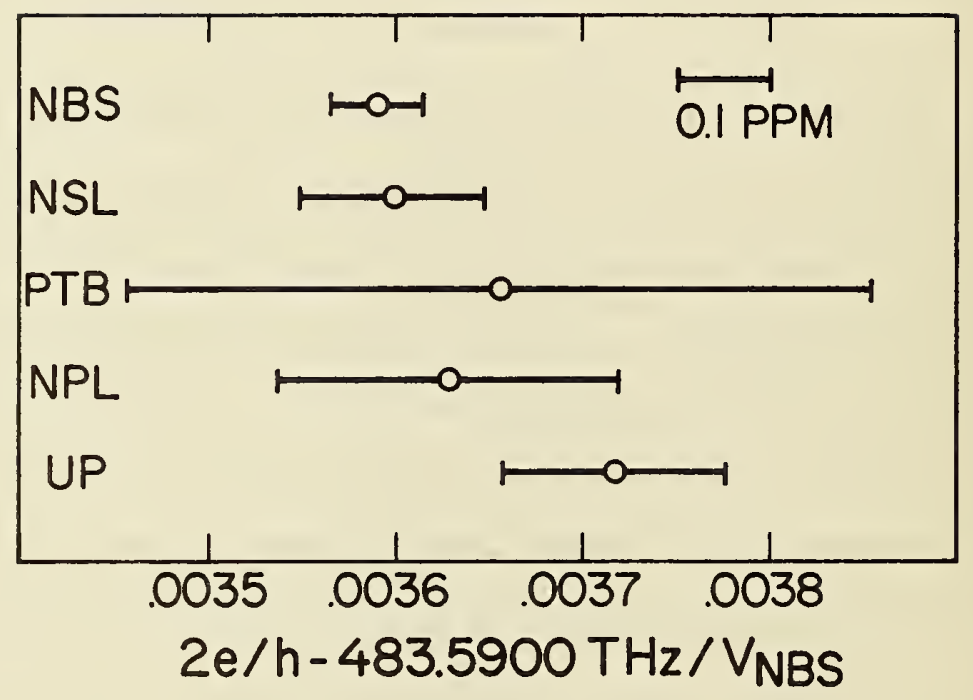

Figure 9. Comparison of measurements of e/h (1971). 


\section{PRECISE METROLOGY}

In this section we collect several applications of superconductivity to precise measurements and measuring instruments. Taken together, they show that this is one area where it does have a natural place. The commercial significance of these applications is the same as for all precise metrology: they exert an important secondary influence, but do not represent a large investment in manufactured hardware.

The Mea surement of e/h

The constant-voltage steps in the current versus voltage characteristic of a Josephson junction, exposed to microwave radiation at frequency $f$, are spaced out at equal intervals $V$, where $V=(h / 2 e) f$. Thus a simultaneous measurement of $V$ and $f$ will yield the value of the fundamental constant $\mathrm{e} / \mathrm{h}$. The constant-voltage steps can be made to extend to about $10 \mathrm{mV}$, permitting a precise measurement with the exercise of some care, while the measurement of the frequency of the microwave source is straightforward.

Finnegan, Denenstein, and Langenberg [58] have performed these measurements to a precision of 3 parts in $10^{8}$. Because of difficulties in relating their maintained standard to the NBS volt, their accuracy was limited to 12 parts in $10^{8}$. While some improvement in accuracy might be worthwhile, it is clear that drift in the primary standard will limit the value of more refined measurements. The measurement of e/h has been accomplished at several standards laboratories including those of the United States [59], Great Britain [60], Australia [61], and West Germany [62]. Results of those measurements, with recent voltage intercomparisons as reference [63], are shown in figure 9. 
Over the course of these measurements the accuracy of the frequency-voltage relation has been probed. Some early concern about frequency pulling effects $[64,65]$ were settled with an article by McCumber [66]. Clark [67] showed experimentally that the frequencyvoltage relation is independent of junction material to at least one part in $10^{8}$. Langenberg and Schrieffer [68] and Hartle, et al. [69] have argued that the relation should be free of quantum electrodynamic corrections. The full impact of the measurement was elucidated by Taylor, Parker, and Langenberg [70] in their monumental reevaluation of the physical constants which was based primarily on the new value for $e / h$.

\section{A New Voltage Standard}

The logical extension of this work is the replacement of the chemical cell by the Josephson effect as our standard emf. Hopefully, within the year, the NBS Electricity Division will base the U.S. legal volt on the Josephson effect rather than the mean value of a group of chemical cells. In practice this will probably be accomplished by using the Josephson effect to monitor and correct the emf of a group of chemical cells at something like two-week intervals.

The agreement on e/h between standards labs of involved nations is quite good and we should expect to eventually see the international volt redefined in terms of the Josephson effect. This would place the volt on the same footing as length and time, both of which are defined in terms of atomic processes.

\section{Absolute Ammeter}

The quantization of magnetic flux in a superconducting ring offers an interesting parallel to the Josephson frequency-voltage relation. If $\mathrm{n}$ flux quanta are contained in the ring then the magnetic flux density, $B$, in the ring is 


$$
B=n \varphi_{0} / S
$$

where $S$ is the ring area and $\varphi_{0}=h / 2 \mathrm{e}$ is the quantum of magnetic flux. Both effects are a result of the macroscopic phase coherence of the superconducting electrons and the fundamental constant ratio e/h enters in a similar fashion. In principle, we need measure only $B$ and $\mathrm{S}$ to obtain a value for $\mathrm{h} / \mathrm{e}$.

Meservey [71] has proposed the construction of an absolute ammeter based upon the above relation. In his proposal, the superconducting ring is replaced by a thin-film SQUID which measures the number of flux quanta enclosed. The field is provided by a carefully characterized solenoid through which the test current is passed. The current is thus measured in terms of the flux enclosed by the SQUID loop, the solenoid geometrical factors, and the area of the SQUID loop. The diagram of figure 10 shows the arrangement.

The concept is sound, but technological problems stand in the way of realization of its full potential. The most severe of these problems is the measurement of the SQUID area and of the solenoid geometrical factors. Present technology places an accuracy limit of the order of one ppm on such measurement. There may be merit in using the scheme to check the consistency of our fundamental constants, but the gains promised as an absolute ammeter are insufficient to overcome the investment of significant effort on such a project. Be mindful that the limitations are largely due to present technology limitations. These limitations may gradually be surmounted and make Meservey's proposal more attractive. The precision of the ammeter far surpasses its accuracy and a later section is devoted to its value as a ratio device. 


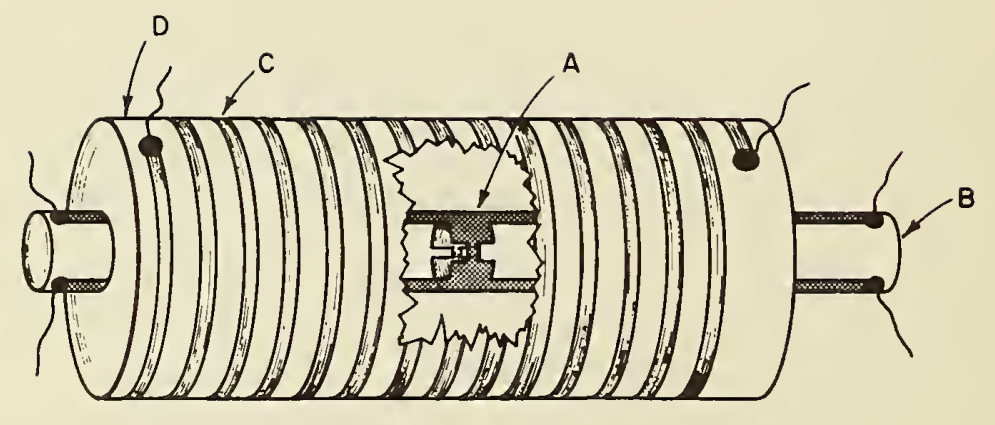

Figure 10. Meservey's proposed absolute ammeter, showing the SQUID (A), and its leads on the quartz rod (B), inside the solenoid (C), supported on the quartz cylinder (D). 
The Measurement of $\mathrm{h} / \mathrm{m}$

If a hollow superconducting cylinder is spun about its longitudinal axis, a field is generated which is just a result of conservation of the canonical momentum of the system. The flux enclosed by the hollow cylinder is [72]

$$
\varphi=\varphi_{0}\left(\mathrm{n}-4 S \omega m e^{/ h}\right)
$$

where $S$ is the area of the cylinder, $m_{e}$ is the electron mass, $h$ is Planck's constant, $\omega$ is the rotation rate, and $n$ is an integer representing the number of flux quanta, $\varphi_{0}$, trapped at zero rotation rate. Parker and Simmonds [72] have used a thin-film SQUID in place of the cylinder to measure the flux generated by rotation and have thus obtained a value for $\mathrm{h} / \mathrm{m}_{\mathrm{e}}$. Their value has an uncertainty of several hundred ppm and is consistent with accepted values of the physical constants. At an uncertainty of $5 \mathrm{ppm}$, this measurement will provide some input to the adjustment of the constants. As with the absolute ammeter, present technology limits the measurement to an uncertainty of the order of one ppm. Field uniformity and alignment problems also place severe restrictions on the measurement.

\section{Frequency Stabilized Oscillators}

The ultrahigh $Q$ cavities developed at Stanford [73] for the superconducting linear accelerator are now finding application as stabilizing elements in microwave oscillators. Benard, et al. [74] at Orsay have used a cavity with an unloaded $Q$ of $10^{9}$ to stabilize an X-band klystron to 3 parts in $10^{12}$ for a period of $10 \mathrm{~s}$. Turneaure and Stein [75] at Stanford have succeeded in obtaining a stability of one part in $10^{13}$ at 10 s and they expect considerable improvement when they achieve better 


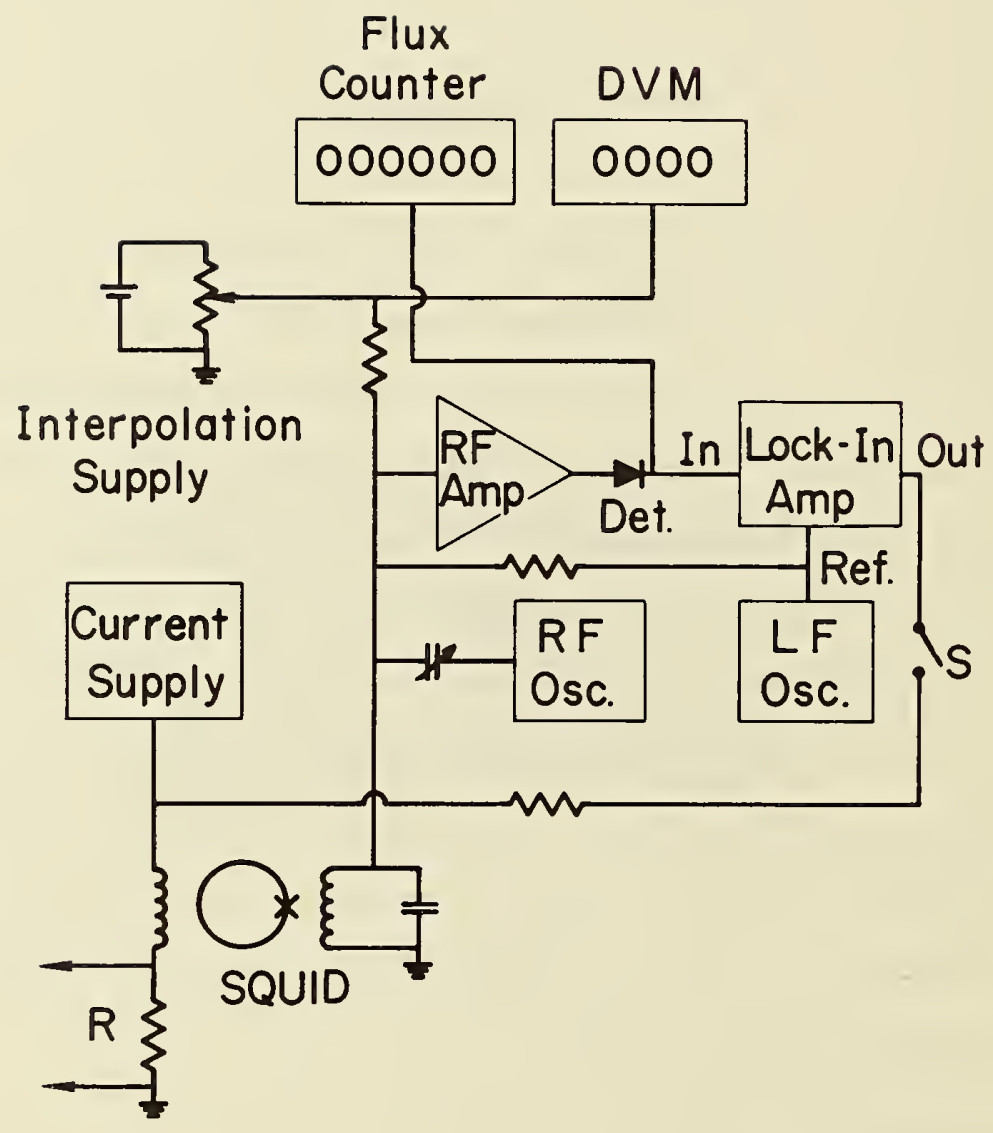

Figure 11. Circuit for precise dc measurements of current or voltage by counting flux quanta. The current supply may be regulated by closing the servo loop at the switch $S$. The instrument basically compares currents, but current ratios may be transformed to voltage ratios by the stable cryogenic resistor $R$. 
temperature stability. If such improvements are realized, these oscillators may well surpass the performance of the temperature-controlled quartz oscillators which are currently used to stabilize klystrons for cesium beam frequency standards. Turneaure suggests that frequency stabilities approaching one part in $10^{16}$ may be feasible.

dc Ratio Measurements

Meservey's ammeter proposal offers an attractive means of obtaining precise current ratios. Towards this end we have been developing a modified version of the ammeter which will be described below. The device should provide an elegant and precise means of measuring current and voltage ratios and furthermore has been demonstrated to be an excellent current regulator. This work is in progress at NBS.

Figure 11 shows a schematic diagram of the system. The interference device is an $\mathrm{rf}$-biased multihole SQUID developed by Zimmerman [22]. The flux state of the SQUID is detected by measuring its radio frequency impedance [18]. The signal detected by the rf circuitry is a periodic function (period of $\varphi_{0}$ ) of the field applied to the SQUID. The current to be measured is passed through the dc coil (solenoid) which is wound about the SQUID. It is clear that the geometrical factors are so complicated that an absolute measurement is prohibited, but for ratio measurements the only requirement is dimensional stability.

The SQUID can be operated in an analog mode [34] by phase detecting the output (modulated by an appropriate signal at the SQUID) and feeding the detected signal back to the rf coil. The system locks onto a peak or valley of the periodic impedance function and the feedback current is then a linear function of the dc field applied to the SQUID. In our system we feed the phase detected signal back to the current supply to be added to the main current delivered by batteries. The SQUID 
then acts to regulate the current through the dc coil. One can thus open the feedback loop and move to any desired flux state and close the loop again to obtain a known current ratio. The ratio of currents in the two states, 1 and 2 , is just $\mathrm{n}_{2} / \mathrm{n}_{1}$ where $\mathrm{n}_{1}$ and $\mathrm{n}_{2}$ are the flux quantum numbers for each of the states (assuming that zero current corresponds with an integral flux state, a condition which can be preset). The flux counter is used to count the number of flux quanta in each state and a small dc current in the rf coil (which produces zero to one flux quantum) facilitates interpolation between flux states.

We can readily interpolate between flux quanta to better than one part in $10^{3}$. If we use $10^{7}$ quanta this gives a dynamic range of $10^{10}$ parts. Reversible or nonreversible motion of flux into the SQUID walls would be the most probable cause of breakdown of the linearity of the device. The same sort of problems are encountered with losses in niobium cavities [75] and these have been handled successfully by careful surface preparation and a high temperature vacuum anneal. Presumably the SQUIDs could be similarly treated should such problems become apparent. So far, we have tested the linearity of this device to $10 \mathrm{ppm}$ over a range of $10^{6}$ quanta. We have regulated a current of $3 \mathrm{~mA}$ to one part in $10^{9}$. We have investigated flux trapping and found a SQUID which appears to be free of this problem at flux level up to $10^{6}$ quanta. More stringent tests are in progress.

This instrument can probably be adapted to a number of other applications. Current ratios could be transformed to voltage ratios through a stable cryogenic resistor (shown in fig. 11). A cryogenic resistor which we have developed $[76,77]$ should allow voltage ratio measurements with a precision commensurate with current ratio measurements. Two coils could be wound in opposition about the SQUID to produce a more sensitive version of the conventional dual arm current 
comparator. This device would be capable of precise current ratio measurements even in the submilliampere range. We plan to investigate such applications as soon as we complete development of the basic instrument.

rf Measurements

The same functions that we have described for dc measurements with a SQUID can be extended to rf measurements also. The first requirement is that the SQUID itself should respond to variations in magnetic flux at the required radio frequency. We have developed such a SQUID [21]. It is an rf-biased device, operating at a frequency of $9 \mathrm{GHz}$. The source of the magnetic flux it senses is the current flowing in a coaxial line inductively coupled to the SQUID. A simple microwave readout system monitors the variation in reflection coefficient of the device in response to variations in current in the coaxial input line.

This prototype device has a flat response to input currents at frequencies from zero to over $1 \mathrm{GHz}$. The readout signal has the functional form $J_{0}\left(2 \pi I / I_{0}\right)$, where $J_{0}$ is the zero order Bessel function, $I$ is the amplitude of an rf current flowing in the input line, and $I_{0}$ is the current required to drive one flux quantum into the SQUID. Figure 12 shows an arrangement to locate the zeros of the Bessel function with a lock-in detector. These provide a natural scale for rf measurements, which can be found in published tables of Bessel functions. The scaling factor $I_{o}$ can be determined by a single measurement with dc.

We have tested the system in a measurement of attenuation at $30 \mathrm{MHz}$ [78]. Over a dynamic range of $40 \mathrm{~dB}$ it was in agreement with the national standard of attenuation in coaxial line to within $\pm 0.004 \mathrm{~dB}$. This is well within the uncertainty normally expressed on calibration 


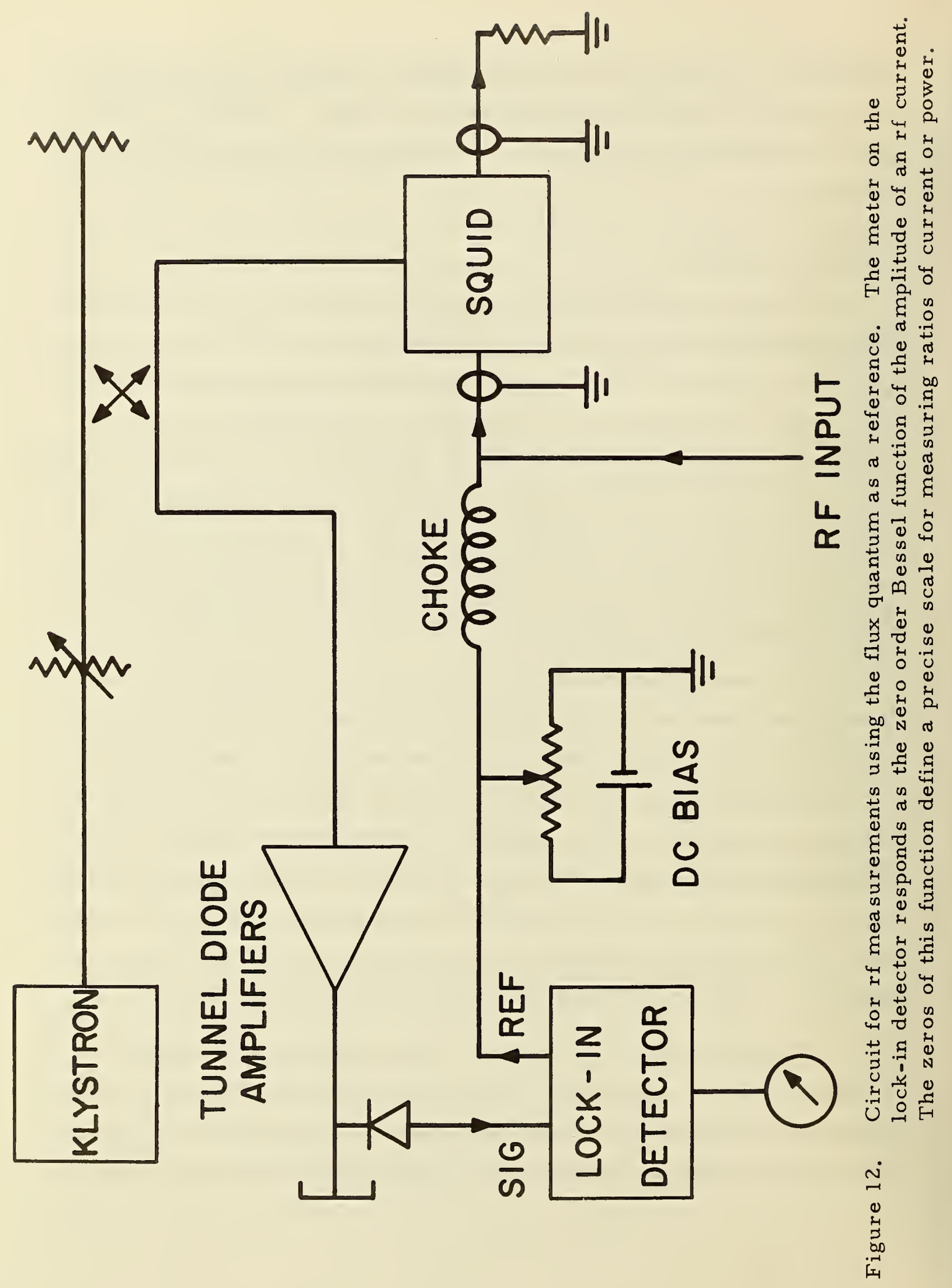


certificates. The overwhelming advantage of the new system is that it is capable of this level of performance at any frequency from zero to $1 \mathrm{GHz}$, whereas the piston attenuator on which the national standard is based is a narrow-band device.

We are presently working to extend the dynamic range of the system by automatic flux quantum counting and by adapting it to calibratable linear detection of very weak signal levels. We have already demonstrated its ability to stabilize an rf current level by using the output of the lock-in detector to control the current through a modulator. Taking advantage of its broadband nature, this system could be used to stabilize the current from a generator with swept frequency. This will support the strong present trend towards radio measurements with swept frequency. In addition to our first measurement of attenuation, the SQUID can be used to measure power, current, and phase difference. It may ultimately replace several of our national rf electrical standards.

Secondary Standards of EMF

There is little question about the value of the Josephson standard of EMF. However, the equipment may not meet the portability and simplicity requirements for laboratory intercomparisons of standards.

For chemical cells during shipment, fractional voltage changes as large as $0.5 \mathrm{ppm}$ are not unusual [79]. For this reason it may be desirable to develop alternate means for transferring and maintaining voltage standards.

Finnegan and Denenstein [79] have suggested such a standard which is based on quasiparticle tunneling between superconductors. The current-voltage characteristic for such a junction if shown in figure 2. The sharp rise in current at the energy gap provides a simple reference EMF. The stability of the bias current on the step need not 
be commensurate with the desired stability in voltage. To operate at an EMF of one volt, approximately 400 such junctions would have to be connected in series. Two distinct advantages which may be realized with this device are: reversibility of the voltage and immunity from adverse effects upon shorting the output.

The SQUID could be used to regulate the current through the tunnel junctions (see the section on dc ratio measurements). This would provide a stability well beyond that necessary for the purpose. In fact, with the current stability offered by this regulator, a stable cryogenic resistor $[76,77]$ might provide an EMF of sufficient stability.

\section{A Low Temperature Voltage Divider}

Perhaps the simplest means of obtaining a precise fixed voltage division with a large ratio is with the series-parallel resistance divider [80]. Switching $N$ matched resistors from series to parallel gives a resistance ratio equal to $\mathrm{N}^{2}$ with an error that is independent, to first order, of the deviations of the individual resistors from their mean value [81]. To obtain a division which is precise to one part in $10^{9}$, the individual resistors need be trimmed to only three parts in $10^{5}$ of the mean value of the set. Because of the resistance of interconnections of the resistors at room temperature, current and voltage compensating resistors must be added if the full value of the concept is to be realized [82]. Such compensated dividers are capable of voltage division with an accuracy of several parts in $10^{8}[58,83]$. The use of superconductors for the resistor interconnections eliminates the need for the compensating resistors. Besides this simplification, cryogenic operation offers the advantages of: low Johnson noise in the resistors (smaller by more than an order of magnitude), negligible thermal EMF's 
and the large thermal conductance of a superfluid helium bath (temperature less than $2.2 \mathrm{~K}$ ) in which the resistors are immersed.

Using a recently developed resistance material [76] we have constructed just such a voltage divider [77]. The instrument was constructed with a conservative design uncertainty of one part in $10^{8}$, although direct tests have only verified this uncertainty to the one ppm level. The resistors (and the ratio of resistance) appear to have at least a short term stability of one part in $10^{8}$.

$\underline{\text { Null Detectors }}$

In conjunction with the divider described above, a SQUID null detector has also been developed [77]. This is essentially the same device as is used for the dc ratio measurements. At $2 \mathrm{~K}$ the null detector has a sensitivity of $5 \times 10^{-12} \mathrm{~A} / \sqrt{ } \mathrm{Hz}$ with an input resistance of $0.1 \Omega$. A number of other devices may be suitable as null detectors, although the input impedance is quite low in some cases. Clarke used a SLUG (Superconducting Low-inductance Undulating Galvanometer) to establish that the Josephson frequency-voltage relation is independent of material to one part in $10^{8}$ [67] Ries and Satterthwaite [84] have developed a superconducting galvanometer based on vibrating coils, while Gifford, et al. [85] and Lukens, et al. [20] describe other variations of the rf-biased SQUID as a galvanometer.

\section{Conclusions}

The foregoing discussions indicate that low temperature devices are making deep inroads into electrical metrology. Unique phenomena as well as low thermal noise appear to offer significant advantages for furthe $r$ applications. The field is one in which a relatively small number of well engineered devices can be expected to have significant impact 
(e.g., the Josephson volt work). The new concepts which are beginning to emerge are perhaps just a hint of a new approach to measurement science.

\section{SPECIALIZED SCIENTIFIC INSTRUMENTS}

This is the "miscellaneous" category required by any honest catalogue. The distinction between this and the previous section is blurred. In general we include here both instruments designed for rather specialized purposes, and working principles which may become instruments with development but are at an early stage yet.

Galvanometers

A large part of the early work on superconducting instruments was devoted to making very sensitive voltmeters. An early example was Templeton's superconducting chopper amplifier [86]. Despite later refinements, this retained enough unsolved problems to prevent its widespread use. One of its descendents was a modulator amplifier developed by Ries and Satterthwaite [84]. This almost came to the stage of being marketed as a picovoltmeter several years ago. It failed because its low input impedance (about $10^{-6} \Omega$ ) limited its useful ness. This was a common weakness of many early superconducting instruments, shared by Clarke's "SLUG" [87], which must be among the simplest of them all. It consisted of a blob of solder frozen onto a niobium wire. At a suitable bias point the contact resistance between the two (with the metals superconducting) is a periodic function of the current in the niobium wire, apparently as the result of a quantum interference effect. The basic sensitivity of this device to current (about $10^{-6} \mathrm{~A} / \sqrt{ } \mathrm{Hz}$ ) is not very impressive: its advantage lies in the extremely low input impedance presented by the short piece of superconducting niobium wire. Making full use of this requires transformer 
coupling [88], but even then it does not appear to compete with null detectors which have been engineered using SQUIDs $[20,77]$.

To summarize: using an rf-biased SQUID it is possible to make a galvanometer with any reasonable desired impedance, and with sensitivity limited by thermal noise (at $4 \mathrm{~K}$ ) generated by the circuit on which measurements are to be made $[20,77]$. With this flexibility, elegant measurements of the electrical and magnetic properties of materials at very low temperatures can be accomplished [85]. The earlier devices with which we opened this section do not offer the same convenience and flexibility.

$\underline{\text { RF Amplifiers }}$

Many superconducting amplifiers operate on the parametric principle [89]. In essence, the power added to the signal by the amplifier is not supplied from a dc power supply, but rather from a "pump" supplying power at some frequency higher than that of the signal, with which it interacts in some non-linear device. The output can be taken either at the same frequency as the input (in a negative resistance parametric amplifier) or at the difference between the pump and signal frequencies (in a parametric up converter). In both cases a substantial power gain is possible with the addition of very little noise. Some remarkably simple devices are possible. Clorfeine [90] and Bura [91] have reported successful parametric amplifiers based on the non-linear penetration of electromagnetic fields through superconducting films. Josephson junctions also work well as the required non-linear devices[92]. The action of the rf-biased SQUID may be described as parametric upconversion, especially when the magnetic flux being sensed varies with time. Zimmerman and Silver $[28,93]$ have explored many of the ramifications of this. Recently, Kanter and Silver [94] have reported early 
results with a parametric (negative resistance) microwave amplifier in which the self oscillation of a Josephson junction under the influence of a voltage bias is used for the pump. The work on noise thermometry which we describe later on in this section demonstrated that parametric amplifiers based on the Josephson effect can have effective noise temperatures less than $10^{-3} \mathrm{~K}$.

In contrast with these parametric amplifiers, Newhouse and Edwards [95] have reported what appears to be a well engineered amplifier based on a cryotron, biased to a point where it is halfway switched. In this condition the gate resistance is a very sensitive function of small variations in control current. It is necessary to use a servo loop to maintain the desired operating point, and the device amplifies at frequencies outside the bandwidth of this servo.

This is an area where spectacular developments could happen very quickly in response to a demonstrated need.

Ultrasonic Acoustics

Tunnel junctions can be used to generate and detect ultrasonic vibrations in the crystal lattice of the substrate [96]. This comes about because of the disturbance of thermal equilibrium during the tunneling process. The major part of the tunneling current at a finite voltage consists of "quasi-particles", or excited electrons not taking part in the superconducting ground state. The sharp rise in current at a voltage of the order of a millivolt reflects the threshold energy required to create these quasi particles. If a junction is biased to a voltage below this threshold, the current can be increased with the assistance of ultrasound to supply the necessary energy. A tunnel junction in this condition therefore becomes a detector of ultrasound. 
When a junction is biased to a voltage over the threshold, a copious current flows. This injects an anomalously large concentration of quasi particles into the positive side of the junction. Equilibrium is restored by the extra quasi particles pairing up and collapsing into the ground state. In this process they radiate most of their energy in the form of ultrasonic waves.

Thus tunnel junctions between superconductors can be used to generate and detect ultrasound at frequencies towards the upper limit of the spectrum of lattice waves in a solid. This is another working principle awaiting a creative application.

\section{Noise Thermometers}

The Josephson effect has been used to extend noise thermometry down to the millikelvin range of temperature [97-101]. The strategy was to use an oscillating (voltage biased) Josephson junction to convert the variations in voltage to be detected into variations in frequency, which can be measured absolutely. A straightforward application of the theory of frequency modulation also eliminated the need to measure the bandwidth of the system. The two major problems of noise thermometry were therefore avoided, and a system was developed which is capable of realizing the absolute scale of temperature with an uncertainty of less than $10^{-3} \mathrm{~K}[101]$. Tests so far have been conducted at temperatures down to $0.02 \mathrm{~K}$.

The principle is illustrated in figure 13. A Josephson junction is connected in a structure of very low inductance $\left(<10^{-9} \mathrm{H}\right)$ with a shunt with much lower resistance than the junction $\left(10^{-5} \Omega\right.$ is a typical value.) A direct current of the order of $10^{-6}$ A (derived from a battery and resistor) through this shunt maintains a bias voltage of about $10^{-11} \mathrm{~V}$, 
which causes the junction to oscillate at a frequency of about $5 \mathrm{kHz}$. The frequency of oscillation $f$ is related to the bias voltage $\mathrm{V}$ by

$$
\mathrm{h} f=2 \mathrm{eV}
$$

where $h$ is Planck's constant and $e$ is the electron charge. The fluctuations in voltage due to thermal noise in the shunt resistor cause variations in the frequency $f$, which may be measured either by using a spectrum analyzer to determine the average linewidth $\Delta f$ (between the points at half peak power) or by using a frequency counter to repeatedly measure the frequency. The quantity of interest is then the variance (or mean square deviation) $\sigma^{2}(f)$ of the fluctuations. The absolute temperature $T$ of the shunt resistor $R$ may then be calculated from the formulae

$$
\begin{gathered}
\Delta f=16 \pi \mathrm{e}^{2} \mathrm{kTR} / \mathrm{h}^{2} \\
\sigma^{2}(f)=8 \mathrm{e}^{2} \mathrm{kTR} / \tau \mathrm{h}^{2}
\end{gathered}
$$

where $\tau$ is the gate-time of the frequency counter.

Equation (9) was verified experimentally [98] in 1967, and our more recent efforts have been directed towards making a practical thermometer based on equation (10). A schematic diagram of this instrument is shown in figure 13. Because of the highly nonlinear characteristics of a Josephson junction, its rf impedance is modulated strongly at the frequency of its self-oscillation. This modulation may be detected at some convenient radio frequency (e.g., $30 \mathrm{MHz}$ ) with the circuit shown. The advantage of this procedure is the substantial power gain available by parametric up-conversion [89]. The readings of the frequency counter are punched onto paper tape and analyzed by a simple computer program. The only additional information required to 
determine absolute temperature is the bias current and the average frequency of oscillation (and hence the bias voltage), from which the value of $R$ in equation 10 may be computed.

This is now at the stage of a working instrument. Soulen [101] is presently engaged in stringent tests and further refinements which will determine the value of noise thermometry as a tool for realizing the Kelvin scale at very low temperatures. Because of the rather long times required for measurements at the lowest temperatures, it probably will not replace the more common secondary thermometers, which require calibration but are capable of the quick readings which are required by routine experimental work. Rival absolute thermometers are based on the temperature dependence of the magnetic susceptibility of nucleii. This can be monitored either by measuring the anisotropy of $\gamma$-ray emission from decaying nuclei [102] or by direct magnetic measurement with a SQUID [103,104]. Recently, Wheatley et al [85] have developed a variant of the noise thermometer, in which the resistor is coupled to an rf-biased SQUID via a transformer. The advantage of this system is that it can use a wider bandwidth than the version we have described here, and hence make measurements with equal precision in a shorter time. However, in doing this the directness of absolute thermometry is sacrificed. Wheatley's thermometer will probably be used in practice with a calibration at a reference temperature determined by some other means. This is common practice in noise thermometry at higher temperatures with conventional techniques [105]. 


\section{TRANSMISSION LINES FOR INFORMATION}

The most efficient way to use the capacity of a given electrical channel for communication is by Pulse Code Modulation (PCM). Thus the merits of transmission lines for information are determined by their behavior with very short pulses. The historical sequence of development started with the investigation of miniature superconducting coaxial lines for delaying pulses. As a result of this work their virtues for the transmission of information were recognized, and this has motivated a further effort to develop them for that purpose. It now appears that they will probably be the most economical way of constructing systems with very large capacity.

The leading pioneers in this field were Nahman and his coworkers. At an early stage they constructed a fairly crude $50 \Omega$ coaxial line with a niobium inner conductor, teflon dielectric (about $2 \mathrm{~mm} \mathrm{OD}$ ), and lead outer conductor. With this they demonstrated the possibilities of superconducting lines for transmitting pulses $[106,107]$. They were able to preserve a risetime of less than $5 \times 10^{-10} \mathrm{sec}$ for a pulse travelling several hundred meters (a delay of several $\mu \mathrm{sec}$ ). Encouraged by this early work, they undertook a detailed analysis of the theoretically attainable performance of superconducting lines $[108,109]$. This showed that there was considerable scope for improvement in the prototype lines.

The factors which limit the transmission of pulses are losses in the conductors and the dielectric, and reflections caused by irregularities in the line. The reason for the superiority of superconducting lines is that the surface resistance of all normal metals, at the frequencies excited by the passage of a pulse, is high because of the anomalous skin effect, regardless of the quality of the metal. Nahman's first crude lines reaped a large part of the available benefit of superconductors 
in this respect. The aspect where most improvement is possible is in the elimination of spurious reflections. This requires manufacture to very tight tolerances. Vigorous development is now in progress in Japan. Hoshiko and Chiba have sketched out their ultimate hopes for an information system using superconducting lines [110]. They envision a cable of 200 individual coaxial lines (each about $1 \mathrm{~mm}$ in diameter), requiring repeaters at intervals of 25 to $50 \mathrm{~km}$. With a bandwidth of $3 \mathrm{GHz}$, the capacity of such a system for transmitting information will be about $300 \mathrm{G}$ bits/sec. In private communications (to Nahman), they report considerable progress towards solving the problem of manufactur ing the cable with the required toler ances.

The nearest conventional rival is a cylindrical waveguide with helical walls to prevent mode mixing. Made with normal metal it could have a bandwidth of $40 \mathrm{GHz}$ - wider than miniature coaxial line. However the attenuation would be greater, and repeaters would be required at intervals of $16 \mathrm{~km}$. These would be the most expensive parts of the system.

This appears to be yet another area where superconductors will have a clear superiority over other technologies when the world requires systems with sufficiently large capacity. 


\section{ACCELEROMETERS AND GYROS}

The combination of the Meissner effect and an inhomogeneous magnetic field, generated by superconducting coils carrying persistent currents, has obvious appeal as a stable bearing with very little friction. The two applications of this principle which have received attention so far are gyros and gravimeters. At the time of writing, the latter appear to be the nearer to acceptance as valuable instruments.

Gyros

The earliest public discussion of the development of a practical superconducting gyro was by Harding and Tuffias [111] in 1960. They listed most of the expected problems and laid out a program of research to solve them. Four years later, Harding reported significant progress but not the completion of the project [112]. One of the major discoveries of the intervening years had been the full extent of ac loss in superconductors, even with well prepared surfaces exposed to modest magnetic fields. This proved to be a severe but not insurmountable problem: superconducting gyros could become very good, but they would never be frictionless. Other groups were working in this field at the same time, but very little of their work was published. The observed conclusion is that superconducting gyros are not in general use for navigation today.

One situation where the problems of superconducting (or any other)gyros are drastically reduced is on board a satellite in orbit. The absence of a strong force of gravitation reduces the restoring forces required of the suspension, and hence gives greater freedom to reduce friction. Fairbank and his students are in the process of developing a gyro with that in mind [113]. Their objective is to detect a small precession (from 0.05 to 7 seconds of arc per year) predicted by the general theory of relativity. They have two possible designs in mind. The first is similar to the levitated spheres that other groups have been working 
with. The second relies on the very long relaxation time of the spinning nuclei of liquid ${ }^{3} \mathrm{He}$. In the absence of external perturbation (which can be excluded by a superconducting shield) the magnetization of these nuclei would persist for several years. Both these designs expect to use a superconducting magnetometer for readout of the direction in which the gyro points. The accuracy required for the experiment is 0.001 seconds. of arc.

Thus some promise remains for the future of superconducting gyros. Meanwhile inertial navigation continues to tolerate the uncertainties of mechanic al suspensions.

Gravimeters

Measurement of long-term variations in the Earth's gravitation requires an elastic suspension which is not subject to the creep that affects metal springs. Prothero and Goodkind have developed an instrument, based on a levitated superconducting body, which satisfies this requirement [114]. It consists of a superconducting sphere levitated by an inhomogeneous magnetic field, maintained by persistent currents in a set of superconducting coils. The position of the sphere is sensed by two independent systems: a magnetic flux detector and a detector which senses the capacitance between the sphere and a set of nearby plates. A signal from the capacitance detector drives a servo system to maintain the sphere in a constant position, by applying a small additional restoring force. By monitoring both position detectors it is possible to discriminate between variations in gravitation and variations in the magnetic field. The stability of the system is at least $10^{-10}$ per hour, and runs of many months are routinely in progress. This instrument has been in operation for several years now, generating information of real geophysical importance [115]. 


\section{CONCLUSION}

The general impression left by this sketch of the present state of cryoelectronics is one of vigorous development, which is beginning to be guided towards the solution of real problems. Shortage of space has precluded doing full justice to a large amount of early work which has since been eclipsed by the research it helped to stimulate. 
References

[ 1] Josephson, B. D., Possible new effects in superconductive tunneling, Phys. Letters $1,251-253$ (July, 1962).

[ 2] Josephson, B. D., Coupled superconductors, Rev. Mod. Phys. 36, 216-220 (January, 1964).

[ 3] Josephson, B. D., Supercurrents through barriers, Advances in Physics 14, 419-451 (October, 1965).

[ 4] Giaever, I., Energy gap in superconductors measured by electron tunneling, Phys. Rev. Letters 5, 147-148 (August, 1960).

[ 5] Clarke, J., Experimental comparison of the Josephson voltagefrequency relation in different superconductors, Phys. Rev. Letters 21, 1566-1569 (December, 1968).

[ 6] Anderson, P. W., and Dayem, A. H., Radio frequency effects in superconducting thin film bridges, Phys. Rev. Letters $\underline{13}$, 195-197 (August, 1964).

[ 7] Zimmerman, J. E., Cowen, J. A., and Silver, A. H., Coherent radiation from voltage biased weakly connected superconductors, Appl. Phys. Letters 9, 353-355 (November, 1966).

[ 8] Nisenoff, M., Thin film superconducting weak link devices, Applied Superconductivity Conference, Annapolis, Maryland (May, 1972). Proceedings to be published by IEEE.

[ 9] Matisoo, J., Josephson tunnel junctions, Applied Superconductivity Conference, Annapolis, Maryland (May, 1972). Proceedings to be published by IEEE.

[10] Zimmerman, J. E., Properties and applications of superconducting point contacts, Applied Superconductivity Conference, Annapolis, Maryland (May, 1972). Proceedings to be published by IEEE. 
[11] Clarke, J., Low frequency measurements with Josephson devices, Applied Superconductivity Conference, Annapolis, Maryland (May, 1972). Proceedings to be published by IEEE.

[12] McCumber, D. E., Effect of ac impedance on dc voltage-current characteristics of superconductor weak link junctions, J. Appl. Phys. 39, 3113-3118 (June, 1968).

[13] Stewart, W. C., Current-voltage characteristics of Josephson junctions, Appl. Phys. Letters 12, 277-280 (April, 1968).

[14] Hansma, P. K., Rochlin, G. I., and Sweet, J. N., Externally shunted Josephson junctions: generalized weak links, Phys. Rev. B ㄴ, 3003-3014 (November, 1971).

[15] Rowell, J. M. , Magnetic field dependence of the Josephson tunnel current, Phys. Rev. Letters 11, 200-202 (September, 1963).

[16] Zimmerman, J. E., and Silver, A. H., Macroscopic quantum interference effects through superconducting point contacts, Phys. Rev. 141, 367-375 (January, 1966).

[17] Silver, A. H., and Zimmerman, J. E., Quantum states and transitions in weakly connected superconducting rings, Phys. Rev. 157, 317-341 (May, 1967).

[18] Zimmerman, J. E., Thiene, P., and Harding, J. T., Design and operation of stable rf-biased superconducting point-contact quantum devices, and a note on the properties of perfectly clean metal contacts, J. Appl. Phys. 41, $1572-1580$ (March, 1970).

[19] Mercereau, J. E., Superconducting magnetometers, Rev. Phys. Appl. 5, 13-20 (February, 1970).

[20] Lukens, J. E., Warburton, R. J., and Webb, W. W., Versatile superconducting femtovolt amplifier and multimeter, J. Appl. Phys. 42, 27-30 (January, 1971). 
[21] Kamper, R. A., and Simmonds, M. B., Broadband superconducting quantum magnetometer, Appl. Phys. Letters 20, 270-272 (April, 1972).

[22] Zimmerman, J. E., Sensitivity enhancement of superconducting quantum interference devices through the use of fractional-turn loops, J. Appl. Phys. 42, 4483-4487 (October, 1971).

[23] Zimmerman, J. E., and Frederick, N. V., Miniature ultrasensi-. tive superconducting magnetic gradiometer and its use in cardiography and other applications, Appl. Phys. Letters 19, 16-19 (July, 1971).

[24] Silver, A. H., and Zimmerman, J. E., Josephson weak link devices, Air Force Report SAMSO-TR-71-18 (November, 1970).

[25] Shapiro, S., Josephson currents in superconducting tunneling: the effect of microwaves and other observations, Phys. Rev. Letters 11 , 80-82 (July, 1963).

[26] Langenberg, D. N. Scalapino, D. J., Taylor, B. N., and Eck, R., Investigation of microwave radiation emitted by Josephson junctions, Phys. Rev. Letters 15, 294-297 (August, 1965).

[27] Yanson, I. K., Svistunov, V. M., and Dmitrenko, I. M., Experimental observation of the tunnel effect for Cooper pairs with the emission of photons, Soviet Physics JETP 21, 650-652 (September, 1965).

[28] Zimmerman, J. E., and Silver, A. H., A high sensitivity superconducting detector, J. Appl. Phys. 39, 2679-2682 (May, 1968).

[29] Ulrich, B. T., and Kluth, E. O., Josephson junction one millimeter microwave source: coupling outside a dewar, Applied Superconductivity Conference, Annapolis, Maryland (may, 1972). Proceedings to be published by IEEE. (See also a post-deadline paper by R. K. Elsley at the same conference.) 
[30] McDonald, D. G., Kose, V. E., Evenson, K. M., Wells, J. S., and Cupp, J. D., Harmonic generation and submillimeter wave mixing with the Josephson effect, Appl. Phys. Letters 15, $121-122$ (August, 1969).

[31] Vant-Hull, L. L., and Mercereau, J. E., Normal Josephson junctions and quantum coherence, Phys. Rev. Letters 17, 629-631 (September, 1966).

[32] Opfer, J. E., Modulated inductance magnetometers, Rev. Phys. App1. 5, 37-40 (February, 1970).

[33] Deaver, B. S., and Goree, W. S., Some techniques for sensitive magnetic measurements using superconducting circuits and magnetic shields, Rev. Sci. Instr. 38, 311 -318 (March, 1967).

[34] Forgacs, R. L., and Warnick, A., Digital-analog magnetometer utilizing superconducting sensor, Rev. Sci. Instr. 38, 214-220 (February, 1967).

[35] Zimmerman, J. E., and Frederick, N. V., Ultrasensitive super conducting magnetic gradiometer, NBS Report 10736 (March, 1972).

[36] Baule, G. M., and McFee, R., Detection of the magnetic field of the heart, Amer. Heart J. 66, 95-96 (July, 1963). See also McFee, R., and Baule, G. M., Research in electrocardiography and magnetocardiography, Proc. IEEE 60, 290-321 (March, 1972).

[37] Cohen, D., Edelsack, E. A., and Zimmerman, J. E., Magnetocardiograms taken inside a shielded room with a superconducting point-contact magnetometer, Appl. Phys. Letters 16, 278-280 (April, 1970).

[38] Rosen, A., Inouye, G. T., Morse, A. L., and Judge, D. L., Magnetic recordings of the heart's electrical activity with a cryogenic magnetometer, J. Appl. Phys. 42, 3682-3684 (September, 1971). 
[39] Cohen, D., Norman, J. C., Molokhia, F., and Hood, W., Jr., Magnetocardiography of direct currents. S-T segment and baseline shifts during experimental mycardial infraction, Science 172, 1329-1333 (June, 1971).

[40] Cohen, D., Magnetoencephalography: evidence of magnetic fields produced by alpha-rhythm currents, Science 161, 784-786 (August, 1968).

[41] Buck, D. A., The cryotron--a superconductive computer component, Proc. IRE $44,482-493$ (April, 1956).

[42] Proc. IEEE 52, no. 10, special issue on cryogenic electronics (October, 1964).

[43] Matisoo, J., The tunneling cryotron--a superconductive logic element based on electron tunneling, Proc. IEEE 55, 172-180 (February, 1967).

[44] Zappe, H. H. , and Grebe, K. R., Ultra high speed operation of Josephson tunneling devices, IBM Journal of Research 15 , 405-407 (September, 1971).

[45] Anacker, W., Potential of superconductive Josephson tunneling technology for ultrahigh performance memories and processors, IEEE Trans. Magnetics MAG-5, 968-975 (December, 1969).

[46] Grimes, C. C., Richards, P. L., and Shapiro, S., Josephson effect far infrared detector, J. Appl. Phys. 39, 3905-3912 (July, 1968).

[47] Grimes, C. C., and Shapiro, S., Millimeter wave mixing with Josephson junctions, Phys. Rev. 169, 397-406 (May, 1968).

[48] Low, F. J., Low temperature germanium bolometer, J. Opt. Soc. Amer. 51, 1300-1304 (November, 1961). 
[49] Martin, D. H., and Bloor, D., The application of superconductivity to the detection of radiant energy, Cryogenics 1, 159-165 (March, 1961).

[50] Bloor, D., Dean, J. J., Jones, G. O., Martin, D. H., Mawer, P. A., and Perry, C. H., Spectroscopy at extreme infrared wavelengths, Proc. Roy. Soc. (London) A260, 510-522 (March, 1961).

[51] Richards, P. L., and Sterling, S. A., Regenerative Josephson effect detector for far infrared radiation, Appl. Phys. Letters 14, 394-396 (June, 1969).

[52] McDonald, D. G., Evenson, K. M., Wells, J. S., and Cupp, J. D. , High frequency limit of the Josephson effect, J. Appl. Phys. 42, 179-181 (January, 1971).

[53] McDonald, D. G., Risley, A. S., Cupp, J. D., and Evenson, K. M., Harmonic mixing of microwave and far infrared laser radiation using a Josephson junction, Appl. Phys. Letters $\underline{18}$, $162-164$ (February, 1971).

[54] McDonald, D. G., Risley, A. S., Cupp, J. D., Evenson, K. M., and Ashley, J. R., Four hundredth order harmonic mixing of microwave and infrared laser radiation using a Josephson junction and a maser, Appl. Phys. Letters 20, 296-299 (April, 1972).

[55] Ulrich, B. T., Astronomical observations with Josephson junction detectors, unpublished invited talk at the Applied Superconductivity Conference, Boulder, Colorado (June, 1970).

[56] Zimmerman, J. E., Heterodyne detection with superconducting point contacts and enhanced heterodyne signals from tightly coupled contacts, J. Appl. Phys. 41, 1589-1593 (March, 1970). 
[57] Blaney, T. G., and Bradley, C. C., The Josephson junction as a frequency analyzer and mixer of submillimeter radiation sources, J. Phys: D $\underline{5}, 180-184$ (January, 1972).

[58] Finnegan, T. F., Denenstein, A., and Langenberg, D. N., ac Josephson effect determination of $\mathrm{e} / \mathrm{h}$ : a standard of electrochemical potential based on macroscopic quantum phase coherence in superconductors, Phys. Rev. B 4, 1487-1522 (September, 1971).

[59] Finnegan, T. F., Witt, T. J., Field, B. F., and Toots, J., $2 \mathrm{e} / \mathrm{h}$ via the ac Josephson effect, Proceedings of the 4 th International Conference on Atomic Masses and Fundamental Constants (September, 1971). To be published.

[60] Petley, B. W., and Morris, K., A measurement of $2 \mathrm{e} / \mathrm{h}$ by the ac Josephson effect, Metrologia 6, 46-51 (April, 1970).

[61] Harvey, I. K., Macfarlane, J. C., and Frenkel, R. B., Deter mination of $2 \mathrm{e} / \mathrm{h}$ based on the ac Josephson effect, Phys. Rev. Letters 25, 853-856 (September, 1970).

[62] Kose, V., Melchert, F., Fack, H., and Schrader, H. -J., Die bestimmung von e/h mit hilfe des Josephson-effektes, PTB Mitteilungen 81, 8-10 (January, 1971).

[63] Finnegan, T. F., Witt, T. J., Field, B. F., and Toots, J., Superconductivity in dc voltage metrology, The Science and Technology of Superconductivity, ed. by W. D. Gregory and W. N. Matthews, 1972, to be published.

[64] Stephen, M. J., Theory of a Josephson oscillator, Phys. Rev. Letters 21, 1629-1632 (December, 1968).

[65] Scully, M. O., and Lee, P. A., Frequency-pulling effects in Josephson radiation, Phys. Rev. Letters 22, 23-26 (January, 1969). 
[66] McCumber, D. E., Frequency-pulling effects in Josephson radiation, Phys. Rev. Letters 23, 1228-1231 (November, 1969).

[67] Clarke, J., Experimental comparison of the Josephson voltagefrequency relation in different superconductors, Phys. Rev. Letters 21, 1566-1569 (December, 1968).

[68] Langenberg, D. N., and Schrieffer, J. R., Comments on quantumelectrodynamic corrections to the electron charge in metals, Phys. Rev. B 3, 1776-1778 (March, 1971).

[69] Hartle, J. B., Scalapino, D. J., and Sugar, R. L., Absence of quantum-electrodynamic corrections to the charge of the electron as measured in Josephson junction experiments, Phys. Rev. B 3 , 1778-1781 (March, 1971).

[70] Taylor, B. N., Parker, W. H., and Langenberg, D. N., The fundamental constants and quantum electrodynamics, Rev. Mod. Phys. 41, 375-496 (July, 1969).

[71] Meservey, R., Proposed ammeter using flux quantization, J. Appl. Phys. 39, 2598-2605 (May, 1968).

[72] Parker, W. H., and Simmonds, M. B., Measurement of h/m e using rotating superconductors, Precision Measurement and Fundamental Constants, NBS Special Publication No. 343, (U.S. Government Printing Office, 1971), pp. 293-248.

[73] Turneaure, J. P., and Viet, N. T., Superconducting Nb TM 010 mode electron-beam welded cavities, Appl. Phys. Letters $\underline{16}$, 333-335 (May, 1970).

[74] Bénard, J., Jimenez, J. J., Sudraud, P., and Viet, N. T., Frequency stability improvement in a klystron stabilized by a superconducting cavity, submitted to Electronics Letters (February, 1972). 
[75] Turneaure, J. P., The status of superconductivity for rf applications, Applied Superconductivity Conference, Annapolis, Maryland (May, 1972). Proceedings to be published by IEEE.

[76] Sullivan, D. B., Resistance of a silicon bronze at low temperatures, Rev. Sci. Instr. 42, 612-613 (May, 1971).

[77] Sullivan, D. B., A low temperature voltage divider and null detector, Rev. Sci. Instr. 43, 499-505 (March, 1972).

[78] Kamper, R. A., Simmonds, M. B., Adair, R. T., and Hoer, C. A., Quantum mechanical measurement of $\mathrm{rf}$ attenuation, Applied Superconductivity Conference, Annapolis, Maryland (May, 1972). Proceedings to be published by IEEE.

[79] Finnegan, T. F., and Denenstein, A., A new transfer and maintenance voltage standard using superconducting tunnel junctions, Metrologia 7, 167 (October, 1971).

[80] Lord Rayleigh, Experiments to determine the value of the British Association Unit of Resistance in absolute measure, Phil. Trans. Roy. Soc. (London) 173, 661-697 (March, 1882).

[81] Wenner, F., Methods, apparatus and procedures for the comparison of precision standard resistors, J. Res. Nat. Bur. Standards 25, 229-294 (August, 1940).

[82] Hamon, B. V., A $1-100 \Omega$ build-up resistor for the calibration of standard resistors, J. Sci. Instr. 31, 450-453 (December, 1954).

[83] Finnegan, T. F., ac Josephson effect determination of e/h: A standard of electrochemical potential based on macroscopic phase coherence in superconductors, PH. D. Thesis, U. of Pennsylvania, 1971. 
[84] Ries, R. P., and Satterthwaite, C. B., Superconducting parametric amplifier for the measurement of small dc voltages, Rev. Sci. Instr. 38, 1203-1209 (September, 1967).

[85] Gifford, R. P., Webb, R. A., and Wheatley, J. C., Principles and methods of low frequency electric and magnetic measurements using an rf-biased point contact superconducting device, J. Low Temp. Phys. 6, 533-610 (March, 1972).

[86] Templeton, I. M., A superconducting modulator, J. Sci. Instr. 32, 314-315 (August, 1955).

[87] Clarke, J., A superconducting galvanometer employing Josephson tunneling, Phil. Mag. 13, 115-127 (January, 1966).

[88] Clarke, J., Tennant, W. E., and Woody, D., Impedance matching a Josephson galvanometer by means of a superconducting transformer, J. Appl. Phys. 42, 3859-3865 (September, 1971).

[89] Louisell, W. H., Coupled Mode and Parametric Electronics (John Wiley and Sons, Inc., New York, 1960).

[90] Clorfeine, A. S., Microwave amplification with superconductors, Proc. IEEE 52, 844-845 (July, 1964).

[91] Bura, P., Parametric amplification with superconducting films, Proc. IEEE 54, 687-688 (April, 1966).

[92] Di Nardo, A. J., and Sard, E., Superconducting microwave mixers utilizing Josephson junctions, (abstract only) J. Appl. Phys. 42, 105 (January, 1971).

[93] Silver, A. H., and Zimmerman, J. E., Multiple quantum resonance spectroscopy through weakly connected superconductors, Appl. Phys. Letters 10, 142-145 (March, 1967).

[94] Kanter, H., and Silver, A. H., Self pumped Josephson parametric amplification, Appl. Phys. Letters 19, 515-517 (December, 1971). 
[ 95] Newhouse, V. L., and Edwards, H. H., An ultrasensitive linear cryotron amplifier, Proc. IEEE 52, 1191 -1206 (October, 1964).

[ 96] Dayem, A. H., Miller, B. I., and Wiegand, J. J., Phonon generation and detection in superconducting lead diodes, Phys. Rev. B $\underline{3}, 2949-2961$ (May, 1971).

[ 97] Kamper, R. A., Millidegree noise thermometry, Symposium on the Physics of Superconducting Devices, Charlottesville, Virginia (April, 1967). Proceedings published as an ONR report. [ 98] Silver, A. H., Zimmerman, J. E., and Kamper, R. A., Contribution of thermal noise to the line width of Josephson radiation from superconducting point contacts, Appl. Phys. Letters 11 , 209-211 (September, 1967).

[ 99] Kamper, R. A., and Zimmerman, J. E., Noise thermometry with the Josephson effect, J. Appl. Phys. 42, 132-136 (January, 1971).

[100] Kamper, R. A., Siegwarth, J. D., Radebaugh, R., and Zimmerman, J. E., Observation of noise temperature in the millikelvin range, Proc. IEEE 59, 1368-1369 (September, 1971).

[101] Soulen, R. J., and Marshak, H., The use of Josephson junctions for noise thermometry below 1 Kelvin, Applied Superconductivity Conference, Annapolis, Maryland (May, 1972). Proceedings to be published by IEEE.

[102] Thorp, T. L., Triplett, B. B., Brewer, W. D., Cohen, M. L., Phillips, N. E., Shirley, D. A., Templeton, J. E., Starky, R. W., and Schmidt, P. H., Search for superconductivity in lithium and magnesium, J. Low Temp. Phys. $\underline{3}$, 589-602 (December, 1970). 
[103] Burhman, R. A., Halperin, W. P., Schwenterly, S. W., Reppy, J., Richardson, R. C., and Webb, W. W., Static nuclear magnetization thermometry in the millikelvin region, Proceedings of the 12 th International Conference on Low Temperature Physics, Kyoto, Japan, 831 -832 (September, 1970).

[104] Goodkind, J. M. , and Stolfa, D. L., The superconducting magnetic flux detector, Rev. Sci. Instr. 41, 799-807 (June, 1970).

[105] Kamper, R. A., Survey of noise thermometry, 5th Symposium on Temperature, Washington, D.C. (June, 1971).

[106] Allen, R. J., and Nahman, N. S., Analysis and performance of superconductive coaxial transmission lines, Proc. IEEE $\underline{52}$, 1147-1154 (October, 1964).

[107] Nahman, N. S., The measurement of baseband pulse rise times of less than $10^{-9}$ second, Proc. IEEE 55, 855-864 (June, 1967).

[108] McCaa, W. D., Jr., and Nahman, N. S., Frequency and time domain analysis of a superconductive coaxial line using the two fluid model, J. Appl. Phys. 39, 2542-2596 (May, 1968).

[109] McCaa, W. D., Jr., and Nahman, N. S., Frequency and time domain analysis of a superconductive coaxial line using the BCS theory, J. Appl. Phys. 40, 2098-2100 (April, 1969).

[110] Hoshiko, S., and Chiba, N., Superconductive communication cables (in Japanese), Oyo Buturi 40, 905-906 (August, 1971).

[111] Harding, J. T., and Tuffias, R. H., The cryogenic gyro, Advances in Cryogenic Engineering 6, 94-105 (conference in August, 1960).

[112] Harding, J. T., Drift data for the cryogenic gyro, Advances in Cryogenic Engineering 10, 137-143 (conference in August, 1964). 
[113] Fairbank, W. M., A superconducting linear accelerator and the use of superconductivity in some fundamental experiments in physics, Symposium on the Physics of Superconducting Devices, Charlottesville, Virginia (April, 1967). Proceedings published as an ONR report.

[114] Prothero, W. A., Jr., and Goodkind, J. M., A superconducting gravimeter, Rev. Sci. Instr. 39, 1257-1262 (September, 1968).

[115] Prothero, W. A., Jr., and Goodkind, J. M., Earth tide measurements with the superconducting gravimeter, J. Geophys. Res. 77, 926-937 (February, 1972). 
U.S. DEPT. OF COMM. BIBLIOGRAPHIC DATA SHEET

1. PUBLICATION OR REPORT NO. NBS-TN-630

2. Gov't Accession

3. Recipient's Accession No.

4. TIT LE AND SUBTITLE

Developments in Cryoelectronics

5. Publication Date

November 1972

6. Performing Organization Code

8. Performing Organization

7. AUTHOR(S)

Robert A. Kamper and Donald B. Sullivan

9. PERF ORMING ORGANIZATION NAME AND ADDRESS

NATIONAL BUREAU OF STANDARDS, Boulder Labs. DEP ARTMENT OF COMMERCE

Boulder, Co. 80302

10. Project/Task/Work Unit No. 2750187

11. Contract/Grant No.

2. Sponsoring Organization Name and Address

13. Type of Report \& Period Covered

Same as Item 9. Survey - 1969 to 1972

14. Sponsoring Agency Code

5. SUP PLEMENTARY NOTES

6. ABSTRACT (A 200-word or less factual summary of most significant information. If document includes a significant bibliography or literature survey, mention it here.)

This is a survey of progress to date in the development of new electronic instruments taking advantage of the unique properties of superconductors.

7. KEY WORDS (AIphabetical order, separated by semicolons)

Electronics; Josephs on Effect; Precise Measurements; Superconductivity

\section{AVAILABILITY STATEMENT}

EX] UNLIMIT ED.

FOR OFFICIAL DISTRIBUTION. DO NOT RELEASE TO NTIS.

\begin{tabular}{|l|c|}
\hline $\begin{array}{l}\text { 19. SECURITY CLASS } \\
\text { (THIS REPORT) }\end{array}$ & 73 \\
UNCL ASSIFIED & NO. OF PAGES \\
\hline $\begin{array}{l}\text { 20. SECURITY CLASS } \\
\text { (THIS PAGE) }\end{array}$ & 22. Price \\
UNCL ASSIFIED & 70 \\
\hline
\end{tabular}





\section{NBS TECHNICAL PUBLICATIONS}

\section{PERIODICALS}

JOURNAL OF RESEARCH reports National Bureau of Standards research and development in physics, mathematics, and chemistry. Comprehensive scientific papers give complete details of the work, including laboratory data, experimental procedures, and theoretical and mathematical analyses. Illustrated with photographs, drawings, and charts. Includes listings of other NBS papers as issued.

Published in two sections, available separately:

\section{- Physics and Chemistry}

Papers of interest primarily to scientists working in these fields. This section covers a broad range of physical and chemical research, with major emphasis on standards of physical measurement, fundamental constants, and properties of matter. Issued six times a year. Annual subscription: Domestic, $\$ 9.50 ; \$ 2.25$ additional for foreign mailing.

\section{- Mathematical Sciences}

Studies and compilations designed mainly for the mathematician and theoretical physicist. Topics in mathematical statistics, theory of experiment design, numerical analysis, theoretical physics and chemistry, logical design and programming of computers and computer systems. Short numerical tables. Issued quarterly. Annual subscription: Domestic, $\$ 5.00$; $\$ 1.25$ additional for foreign mailing.

\section{TECHNICAL NEWS BULLETIN}

The best single source of information concerning the Bureau's measurement, research, developmental, cooperative, and publication activities, this monthly publication is designed for the industry-oriented individual whose daily work involves intimate contact with science and technology-for engineers, chemists, physicists, research managers, product-development managers, and company executives. Includes listing of aIl NBS papers as issued. Annual subscription: Domestic, $\$ 3.00 ; \$ 1.00$ additional for foreign mailing.

\section{Bibliographic Subscription Services}

The following current-awareness and literaturesurvey bibliographies are issued periodically by the Bureau: Cryogenic Data Center Current Awareness Service (weekly), Liquefied Natural Gas (quarterly), Superconducting Devices and Materials (quarterly), and Electromagnetic Metrology Current Awareness Service (monthly). Available only from NBS Boulder Laboratories. Ordering and cost information may be obtained from the Program Information Office, National "Bureau of Standards, Boulder, Colorado 80302 .

\section{NOHPERIODICALS}

Applied Mathematics Series. Mathematical tables, manuals, and studies.

Building Science Series. Research results, test methods, and performance criteria of building materials, components, systems, and structures.

Handbooks. Recommended codes of engineering and industrial practice (including safety codes) developed in cooperation with interested industries, professional organizations, and regulatory bodies.

Special Publications. Proceedings of NBS conferences, bibliographies, annual reports, wall charts, pamphlets, etc.

Monographs. Major contributions to the technical literature on various subjects related to the Bureau's scientific and technical activities.

National Standard Reference Data Series. NSRDS provides quantitative data on the physical and chemical properties of materials, compiled from the world's literature and critically evaluated.

Product Standards. Provide requirements for sizes, types, quality, and methods for testing various industrial products. These standards are developed cooperatively with interested Government and industry groups and provide the basis for common understanding of product characteristics for both buyers and sellers. Their use is voluntary.

Technical Notes. This series consists of communications and reports (covering both other-agency and NBS-sponsored work) of limited or transitory interest.

Federal Information Processing Standards Publications. This series is the official publication within the Federal Government for information on standards adopted and promulgated under the Public Law 89-306, and Bureau of the Budget Circular A-86 entitled, Standardization of Data Elements and Codes in Data Systems.

Consumer Information Series. Practical information, based on NBS research and experience, covering areas of interest to the consumer. Easily understandable language and illustrations provide useful background knowledge for shopping in today's technological marketplace.

\section{CATALOGS OF NBS PUBLICATIONS}

NBS Special Publication 305, Publications of the NBS. 1966-1967. When ordering, include Catalog No. C13.10:305. Price \$2.00: 50 cents additional for foreign mailing.

NBS Special Publication 305, Supplement 1, Publications of the NBS, 1968-1969. When ordering, include Catalog No. CI3.10:305/Suppl. I. Price $\$ 4.50 ; \$ 1.25$ additional for foreign mailing.

NBS Special Publication 305. Supplement 2, Publications of the NBS, 1970. When ordering, include Catalng No. CI3.10:305/Suppl. 2. Price \$3.25: 85 cents additional for forrign mailing. 
U.S. DEPARTMENT OF COMMERCE

National Bureau of Standards

Washington, D.C. 20234

PDSTAGE AND FEES PAID U.S. DEPARTMENT DF CDMMERCE

DFFICIAL BUSINESS

Penalty for Private Use, \$3DD 




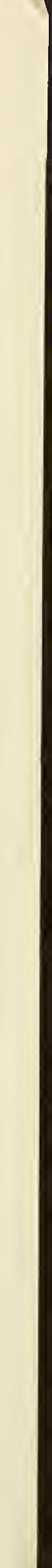





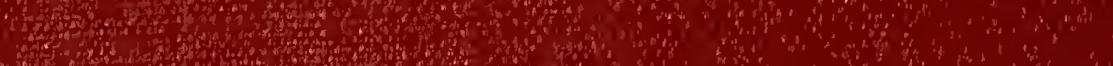

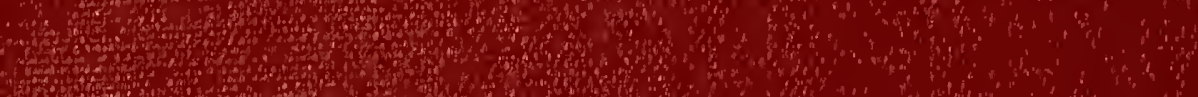

(1)

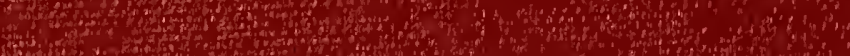

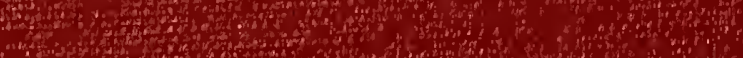

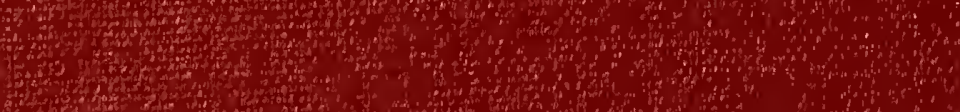

(2)

(2)

(19)

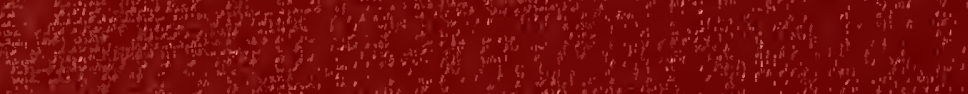

and

20.

60.

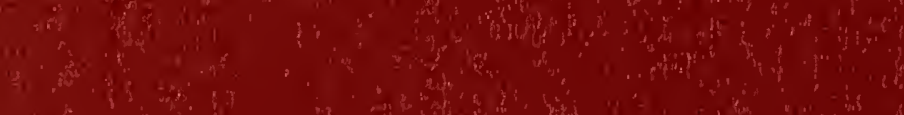

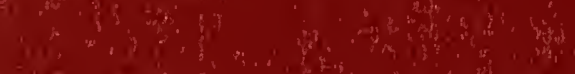

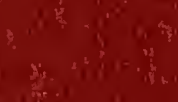

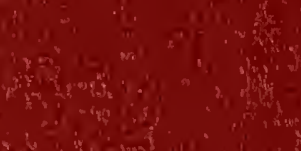

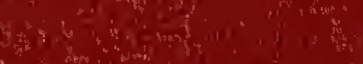

Ant

(1)

Not

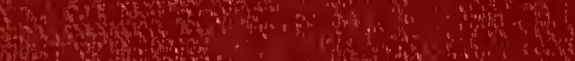

(5)

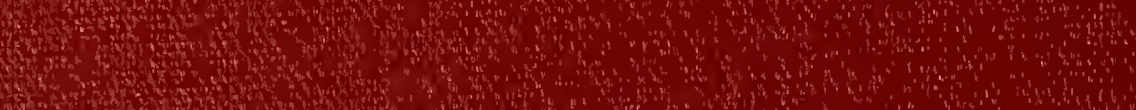
Exte

To

W

(3)

15.

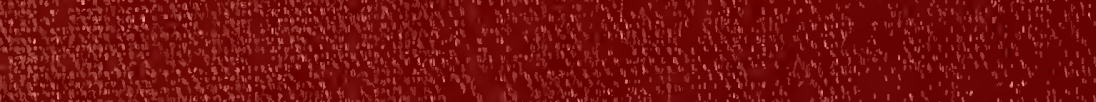

Q

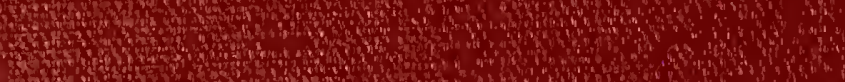

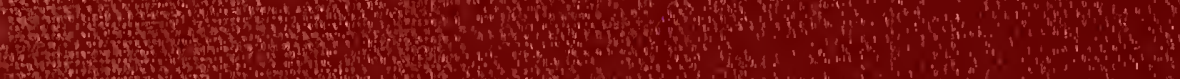

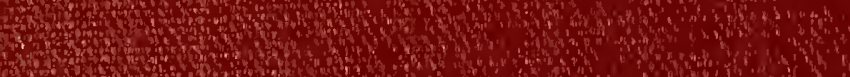
H.

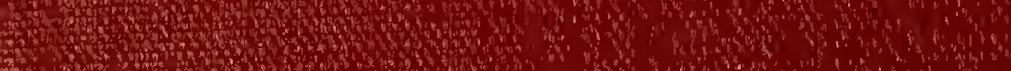

